\title{
Overexpression of Uromodulin-like1 accelerates follicle depletion and subsequent ovarian degeneration
}

\author{
W Wang ${ }^{*, 1}$, Y Tang ${ }^{1}$, L Ni ${ }^{1}$, E Kim ${ }^{1}$, T Jongwutiwes ${ }^{1}$, A Hourvitz ${ }^{2}$, R Zhang ${ }^{3}$, H Xiong ${ }^{3}$, H-C Liu ${ }^{1}$ and Z Rosenwaks ${ }^{1}$
}

Murine Uromodulin-like 1 (Umodl1) encodes $\mathrm{Ca}^{2+}$-dependent EGF-like membrane-bound proteins. This study presents its novel expression in the immune and female reproductive systems. Upon stimulation by CD3/CD28 antibodies, Umodl1 showed a prompt and robust response in the proliferating $\mathrm{CD}^{+}{ }^{+} \mathrm{T}$ cells, suggesting its implication in immune defense against pathogens. In ovary, Umodl1 is regulated by gonadotropins. Mice carrying extra copies of functional Umodl1 were generated by BAC transgenesis. Defects in the female reproductive system became evident from 4 months of age, manifested by reduced or diminished fertility. Histology revealed that the ovaries contained very few discernible follicles in the cortical region, and were devoid of distinguishable corpus lutea (CL). Among the multilayered preantral follicles, elevated apoptosis was observed in both the oocytes and surrounding granulosa cells (GCs). Furthermore, a high level of PPAR $y$ indicated an abnormal adipogenesis in the mutant ovaries, which resulted in the conversion of GCs into adipocytes. By 6 months of age, all mutant mice became anovulatory. Ovarian tissues including $\mathrm{CL}$, follicles of various stages and associated stromal cells were degenerated. Altered expression of AMH, follicle-stimulating hormone and other ovary-specific marker genes such as Gdf-9, Rnf35, NOHLH and Gcx-1 further demonstrated that the molecular properties of the mutant ovaries have been severely disturbed. This work presents a novel animal model for investigating the pathogenesis of premature ovarian failure or early ovarian ageing.

Cell Death and Disease (2012) 3, e433; doi:10.1038/cddis.2012.169; published online 29 November 2012

Subject Category: Experimental Medicine

Reproductive potential in women declines with age. On average, optimal female fertility peaks during the early $20 \mathrm{~s}$ and then deceases sharply after the age of 35 . In humans, the number of germinal cells declines steadily during their embryonic development from seven million primitive oocytes at 20 weeks' gestation to two million at birth. At menarche, there is a further diminution in the follicle pool to $\sim 300000$ among which less than 5000 undergo the follicle maturation processes. Overall, only about 400 oocytes acquire reproductive competence and are ovulated, whereas the majority of follicles degenerate by atresia. ${ }^{1}$ In order to maintain reproductive potential, a woman must have around 25000 follicles remaining in the ovary; women with fewer follicles will show signs of accelerated decline in fecundity, for example, irregular periods, poor response to hormonal stimulation, elevated follicle-stimulating hormone (FSH) and luteinizing hormone (LH) levels and deterioration of oocyte quality. ${ }^{2,3}$ While the number and quality of the remaining oocytes in ovary are closely correlated, the cause(s) of age-related decline of oocyte quality is elusive.

Rodent models have been extensively studied to elucidate the ovarian ageing process as they have similarity to neuroendocrine aspects of human ovarian biology and their reproductive traits can be easily manipulated. Forkhead box O3 transcription factor (Foxo3a) is a member of forkhead transcription factors that may involve in cell cycle arrest, apoptosis and specific stress responses. ${ }^{4}$ More specifically, Foxo3a functions as a suppressor of follicular activation. Foxо3a-/- female mice exhibited premature follicular activation and atresia, thus resulting in early depletion of ovarian reserve. ${ }^{5} \mathrm{FSH}$ exerts its biological functions via its receptors that exclusively reside in the granulosa cells (GCs)

\footnotetext{
${ }^{1}$ The Ronald O. Perelman and Claudia Cohen Center for Reproductive Medicine, Weill Medical College of Cornell University, New York, NY, USA; ${ }^{2}$ IVF Unit, Department of Obstetrics and Gynecology, Chaim Sheba Medical Center, Tel Hashomer, Israel and ${ }^{3}$ Department of Medicine, Immunology Institute, Mount Sinai School of Medicine, New York, NY, USA

${ }^{*}$ Corresponding author: W Wang, The Ronald O. Perelman and Claudia Cohen Center for Reproductive Medicine, Weill Medical College of Cornell University, New York, NY 10065, USA. Tel: + 212746 3187; Fax: + 212746 8996; E-mail: wew2004@ @ed.cornell.edu

Keywords: uromodulin-like 1; BAC transgenesis; ovarian follicle; oocyte; premature ovarian failure; gain-of-function

Abbreviations: APC, antigen-presenting cells; BAC, bacterial artificial chromosome; Bax, BCL-2-associated X protein; Bcl2l10, Bcl-2 like-10 protein; BF, bright field view; BMP-15, bone morphogenic protein-15; Bv, blood vessels; cGC, cumulus granulosa cell; CL, corpus luteum; CS, ovarian cortical stroma; Ctx, thymic cortex; DF, dark field view; eCG, equine chorionic gonadotropin; EGF-Ca ${ }^{2+}$, calcium-binding EGF-like domain; EMI, emilin domain; FN3, fibronectin type III domain; Foxo3, forkhead box $\mathrm{O} 3$ transcription factor; FSH, follicle-stimulating hormone; FSHR, follicle-stimulating hormone receptor; GC, granulosa cell; Gcx-1, granulosa cell specific high-mobility group-box protein-1; GDF-9, growth/differentiation factor-9; Ge, germinal epithelium; $\mathrm{H}$, heart; ISH, in situ hybridization; LH, luteinizing hormone; Me, thymic medulla; mGC, mural graunlosa cell; NOHLH, spermatogenesis- and oogenesis-specific basic helix-loop-helix 1; O, oocyte; OB, olfactory bulb; OC, organ of Corti; Of, ovarian follicle; OG, olfactory glomeruli; ON, olfactory neurons; Ov, ovary; SG, spiral ganglion; PPAR $\gamma$, peroxisome proliferator-activated receptor gamma; Rnf35, ring finger protein 35; SEA, domains found in sea urchin sperm protein Agrin; SP, signal peptide; TC, theca cell; Th, thymus; TM, transmembrane domain; Umodl1, uromodulin-like 1; VNO, vomeronasal organ; WAP, whey acidic protein domain; ZP, zona-pellucida like domain.
}

Received 27.2.12; revised 25.7.12; accepted 21.8.12; Edited by D Aberdam 
in ovary. FSHR - I- females are infertile partly due to the absence of large Graafian follicles and corpora lutea (CL). Other oocyte-specific factors such as growth/differentiation factor-9 (GDF-9) and bone morphogenic protein-15 (BMP-15) may have an autocrine role in regulating oocyte development and maturation, and/or a paracrine role in regulating the proliferation and differentiation of GCs. Mice lacking GDF-9 exhibited a block in follicular development beyond the primary one-layer follicle stage, which leads to complete infertility. ${ }^{6}$ Despite apparently normal folliculogenesis, BMP-15-/mice were subfertile due to defective ovulation. ${ }^{7}$ In contrast, inactivation of the pro-apoptotic gene Bax in mice delayed ovarian ageing likely by granting some protection to the GCs and oocytes against apoptosis. ${ }^{8}$ Collectively, dissecting the molecular mechanism governing the follicle pool and the processes underlying the generation of healthy oocytes will aid in identifying early markers for ovarian ageing and developing therapeutic strategies.

The human uromodulin-like 1 (UMODL1) was first reported and maps to Chromosome 21q22.3, in the minimal critical region likely associated with both trisomy 21 Down's syndrome and congenital high myopia. ${ }^{9-11}$ Notably, some trisomy 21 Down's syndrome patients do display olfactory dysfunction and reduced fertility. ${ }^{12}$ The mouse homolog is preferentially expressed in olfactory and vomeronasal neurons, as well as the sensory epithelial cells of inner ear. ${ }^{13-16}$ Here, we report novel expression data of Umodl1 in thymus and maturing ovarian follicles. To investigate its physiological roles, the gain-of-function approach was employed, by which extra copies of functional Umodl1 were introduced into the mouse genome. Analysis of defects in the reproductive system clearly demonstrates that elevated levels of Umodl1 in vivo accelerate ovarian senescence.

\section{Results}

Expression of endogenous Umodl1. Umodl1 proteins from human and mouse share $58 \%$ identity and $71 \%$ homology in their amino acid composition, and the same patterns in the organization of all conserved domains, including the $\mathrm{Ca}^{2+}$-binding EGF-like, FN3, ZP, SEA and WAP domains (Figure 1a). Serial Analysis of Gene Expression has shown that human UMODL1 is dramatically up-regulated in cancer tissues originated from the lymph node, bladder, liver pancreas and ovary (Figure 1b). In mice, in addition to its presence in olfactory organs and inner ear, ${ }^{13,14}$ novel domains of expression were found in oocytes and thymic medulla (Figures 1c-e). Dual immunofluorescence analysis confirmed that Umodl1 is solely expressed in the CD11c ${ }^{+}$antigen-presenting cells (APCs; Figures 1f-k). Umodl1 protein is normally absent in naïve $\mathrm{CD} 4^{+}-\mathrm{T}$ cells. However, when challenged by anti-CD3/CD28 antibodies, proliferating splenic CD4 ${ }^{+} \mathrm{T}$ cells showed significant levels of Umodl1. Similar up-regulation of Umodl1 was observed in the stimulated thymic TCR $\beta^{+}$T cells (Figure 11 ). To examine the stimulatory effect of gonadotropin on Umodl1 expression, total RNAs from equine chorionic gonadotropin (eCG)primed ovaries were extracted at indicated time intervals and subjected to Northern blot analysis. Substantial increases in Umodl1 mRNA were seen between 8 to $24 \mathrm{~h}$ after the eCG injection, coinciding with the vigorous follicular growth during the transition from preantral to antral stage (Figure $1 \mathrm{~m}$ ). Our expression data suggest a putative role of Umodl1 in mediating cross-talking between the immune and reproductive systems.

Generation of Umodl1-bacterial artificial chromosome (BAC) transgenic ( $\mathrm{Tg})$ mouse lines. The targeting strategy for making the BAC transgene that produces functional Umodl1 proteins is illustrated in Figure $2 \mathrm{a}$. The modified BAC, pW224 was introduced into the mouse genome by pronuclear injection. Four independent lines that carried varying copy numbers of the transgene were established (Figures $2 \mathrm{~b}$ and c). All Tg mice showed a significant increase in Umodl1 expression ranging from 0.6 to 1.2 fold over the wild-type (WT) control as confirmed by quantitative real-time PCR (Q-PCR) (Figure 2d; ${ }^{\star} P<0.05$ ). As identical expression patterns and phenotypes were observed in females from the different founder lines, data presented here were obtained primarily from the pW224-62 Tg line.

Umodl1/lacZ ( $p$ 224) transgene was first detected in the otic vesicle at E10.5 (Supplementary Figure 1a). From E16.5 onwards, many components of inner ear, including the sensory epithelia patches of the vestibule and the sensory hair cells in cochlear duct (arrowheads and arrows, respectively, Figure 3d), were lacZ positive (also Figure 3c). From $\mathrm{E} 13.5$, the basal region of the vomeronasal organ (VNO) and the sensory neurons projected from VNO to the accessory olfactory bulb became positive for pW224 (Figures 3a, b, i and j). In young adult mice, sensory epithelial cells in the nasal cavity and VNO were actively expressing pW224 (Figure 3i; Supplementary Figure 1c). In olfactory bulb, axonal cells in the targeted glomeruli showed strong expression (Figures $3 \mathrm{k}$ and I; Supplementary Figure 1b). Umodl1 is one of the earliest

Figure 1 Spatial and temporal expression profile of the endogenous mouse and human Umodl1 genes. (a) Schematic comparison of functional domains between mouse and human Umodl1 proteins. (b) Differential expression of human UMODL11 in normal and cancer tissues examined by Serial Analysis of Gene Expression (SAGE; adapted from http://www.genecards.org/cgi-bin/carddisp.pl?gene=Umodl; The SAGE analysis is accomplished by a joint effort by the Weizmann Institute of Science, the Salk Institute for Biological Studies and Tufts University ). (c-e) Umodl1 mRNA distribution detected by ISH. Paraffin sections of WT mouse tissues were tested with either ${ }^{35} \mathrm{~S}-$ or digoxigenin-labeled riboprobes. Umodl1 signal was visualized by autoradiography (c and d) or alkaline phosphatase staining (e), respectively. $\mathbf{c}$ is the bright field view of the section in $\mathbf{d}$. (f-k) Immunofluorescence showing Umodl1 in thymus. $\mathbf{f}$ and $\mathbf{g}$ are the same section dual-labeled with TCR $\beta$ and Umodl1. $\mathbf{h}$ is the merged $\mathbf{f}$ and $\mathbf{g}$. (i-k) Dual immunofluorescence analysis of CD11c (i) and Umodl1 (j) co-expression in the APCs. $\mathbf{k}$ is the merged image of $\mathbf{i}$ and $\mathbf{j}$. (I) Western blot analysis showing stable translation of Umodl1 in the proliferating splenic CD4 ${ }^{+} \mathrm{T}$ cells and TCR $\beta^{+}$thymocytes once stimulated by anti-CD3/CD28 antibodies. Untreated splenic CD4 ${ }^{+} \mathrm{T}_{\text {cells }}$ and TCR $\beta^{+}$ thymocytes were included as the negative controls. $\beta$-actin was used as the internal loading control. $(\mathbf{m})$ Northern blot showing the time course of mouse Umodl1 expression in vivo after gonadotropin stimulation. Size of the scale bars is indicated in the corresponding section. The asterisks show the human cancerous tissues in which Umodl1 is drastically up-regulated. 


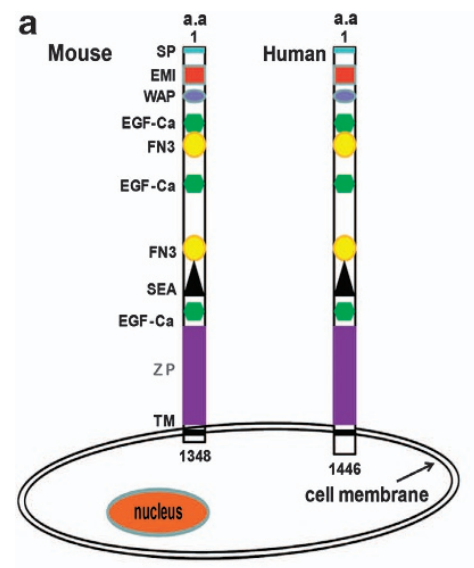

b

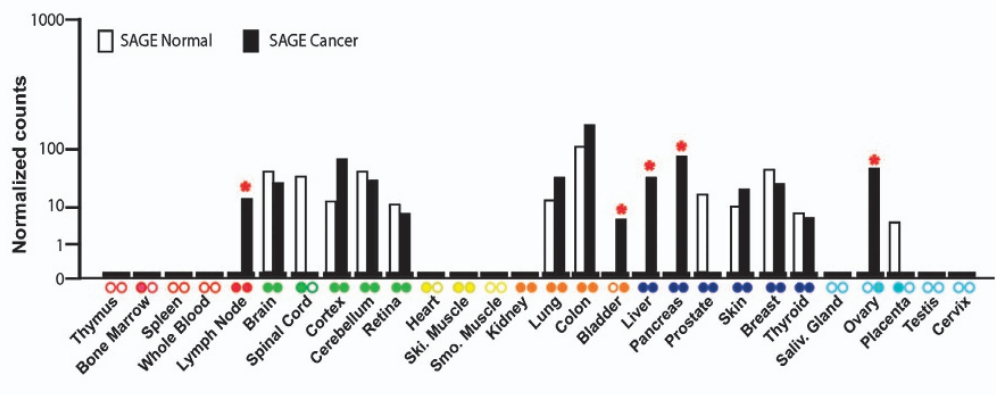

Ovary

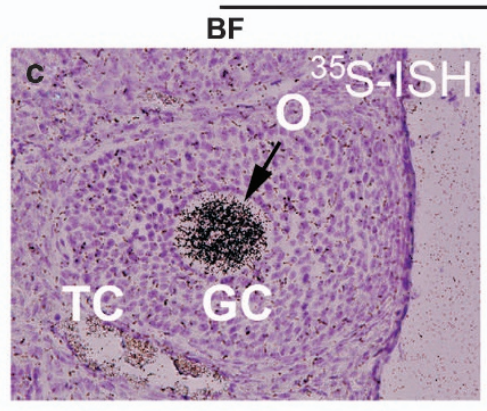

DF

Thymus
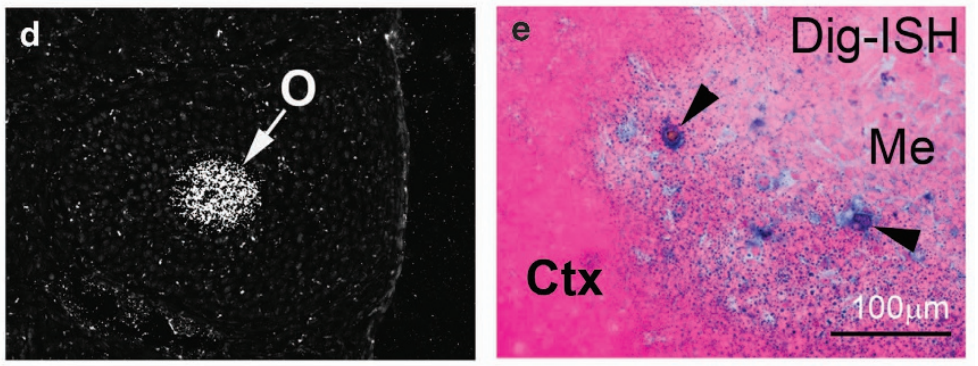

WT Thymus
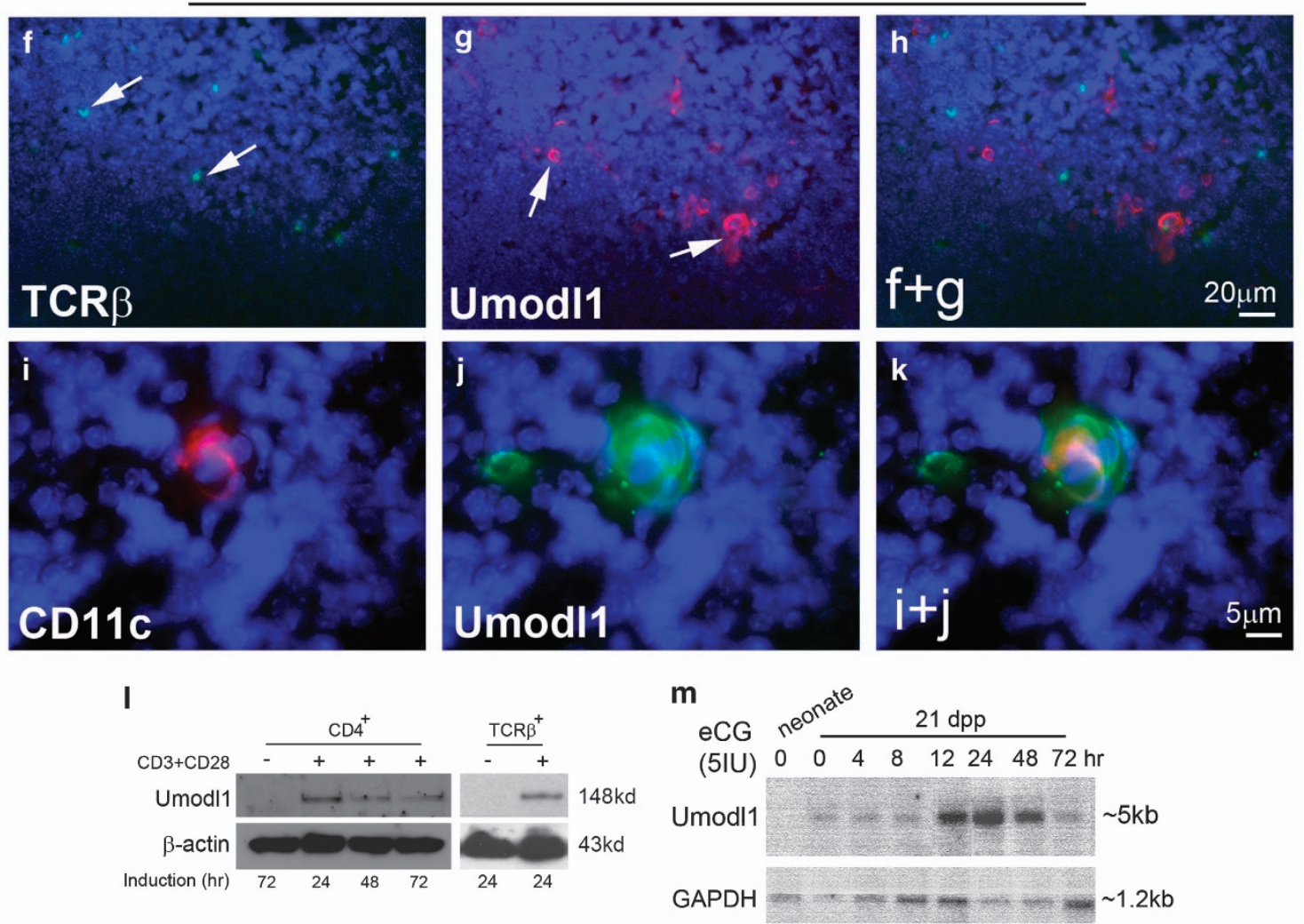
a

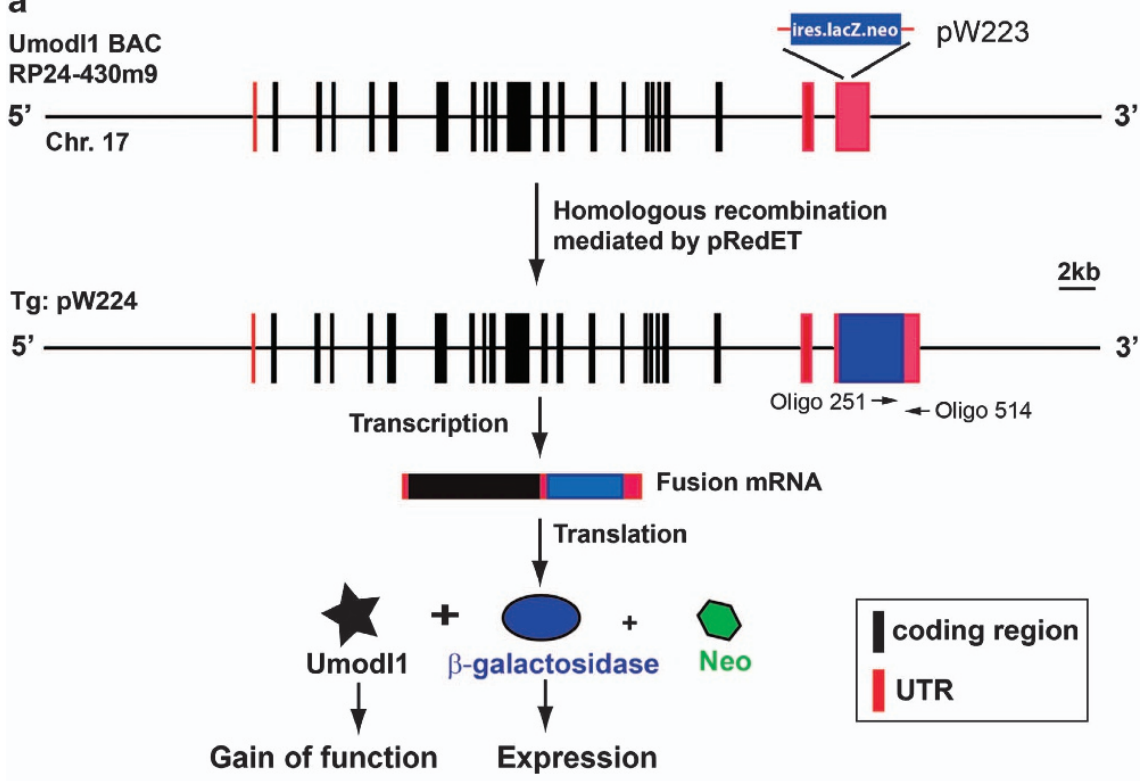

b
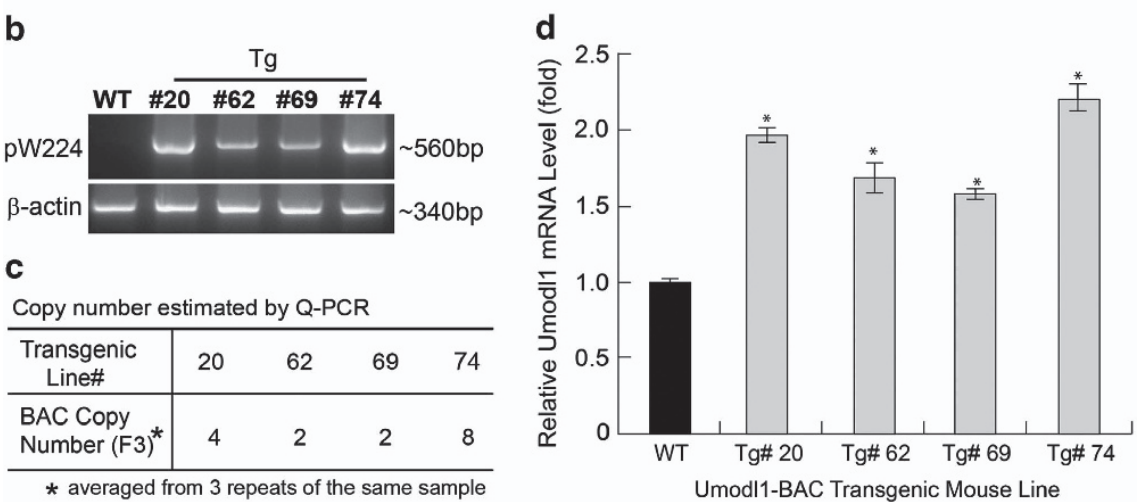

Figure 2 Construction and characterization of the Umodl1 BAC transgene. (a) The size and position of RP24-430m9 Umodl1 BAC are indicated. Linearized targeting vector, pW223, was used to modify the BAC. Mouse Umodl1 is comprised of 22-24 exons that spread over $56 \mathrm{~kb}$ in length on chromosome 17, and produces four alternative splicing transcripts. The BAC clone RP24-430m9 contains a $176 \mathrm{~kb}$ genomic fragment that harbors the entire Glp1rand Umodl1, as well as the first three exons of $A b c g 1 a t$ the $3^{\prime}$ end. This BAC clone was modified by inserting the reporter ires.lacZ.GT1.2/neo into the $3^{\prime}$-UTR of Umodl1. A unique Notl restriction site located in the 4th intron of G/p1r was utilized to inactivate Glp1r. From the resultant pW224, functional Umodl1, $\beta$-galactosidase and neomycin phosphotransferase can be produced. Tg mice were generated by pronuclear injection of Notl-digested pW224. (b) Stable pW224 Tg lines were verified by genomic PCR using Oligo 251 and Oligo 514. (c) Copy number of each Tg line was estimated by the Taqman real-time PCR method. (d) Quantitative PCR to compare Umodl1 mRNA expression in the olfactory bulbs of different Tg lines to the WT control. GAPDH mRNA was amplified in parallel and used as the quantity control. Data were obtained from three independent repeats. ${ }^{*} P<0.05$

transcribed genes in developing thymus. lacZ activity was detected in the thymic primordium as early as E10.5 (Supplementary Figure 1a), and reached its highest level at E16.5 (Figures 3a, e and f). Once the mice reached puberty, pW224 was down-regulated and restricted to the APCs in thymus (Figures $3 \mathrm{~m}$ and $\mathrm{n}$ ). pW224 was detectable above background levels in the interstitial cells of embryonic ovaries (arrowheads, Figures $3 \mathrm{~g}$ and h). All embryonic primordial follicles were negative for Umodl1 at this time (arrows, Figure 3h). From postnatal day 12, oocytes in the advanced follicles ( $>$ primordial stage) were robustly expressing $p W 224$ (Figures 30 and p).

An identical pattern of $p W 224$ expression relative to the WTUmodl1 demonstrates that the modified transgene contains all of the regulatory elements required for recapitulating the endogenous Umodl1 expression. Furthermore, elevated
Umodl1 levels in the $\mathrm{Tg}$ mice indicate that insertion of the reporter into the $3^{\prime}$-UTR poses little/no effects on the transcription/translation of Umodl1. Therefore, Tg mice carrying extra copies of Umodl1 were successfully generated.

Umodl1 is a gonadotropin-responsive gene. Subtractive cloning of Umodl1from ovaries, together with its differential expression in immune cells, collectively demonstrate that Umodl1 is inducible in response to different stimuli. A notably higher level of lacZ activity was observed in eCG-treated Tg ovaries than that from the non-stimulated controls (Figures $4 a$ and $b$ ). In maturing follicles, eCG stimulation resulted in ectopic expression of pW224 in the cumulus cells (Figures 3c and $d$ ). The stimulatory effect of gonadotropin was further quantified by western blot assay and densitometry. The basal level of Umodl1 (without eCG) in the Tg oocytes is about $60 \%$ 
E16.5
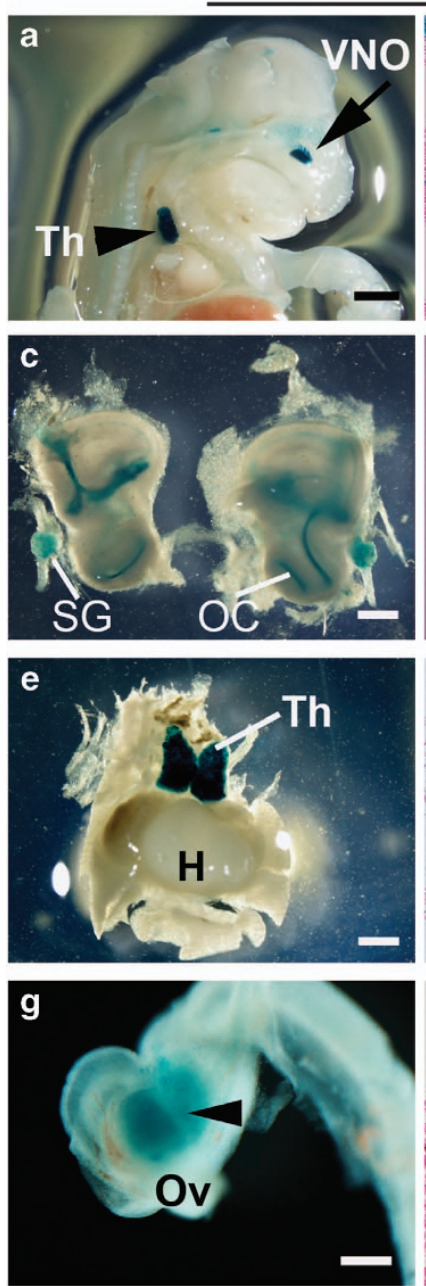
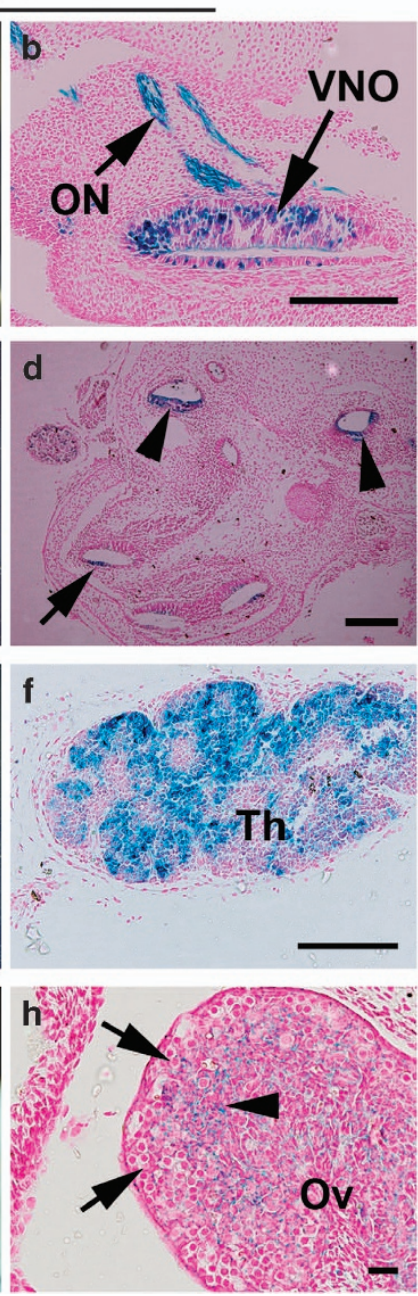

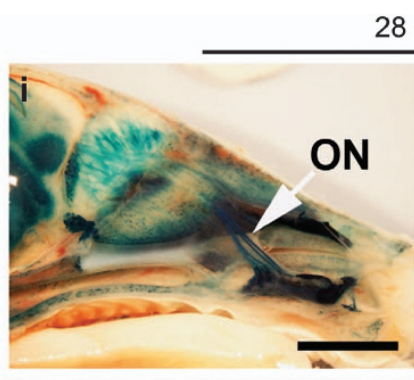

$28 \mathrm{dpp}$
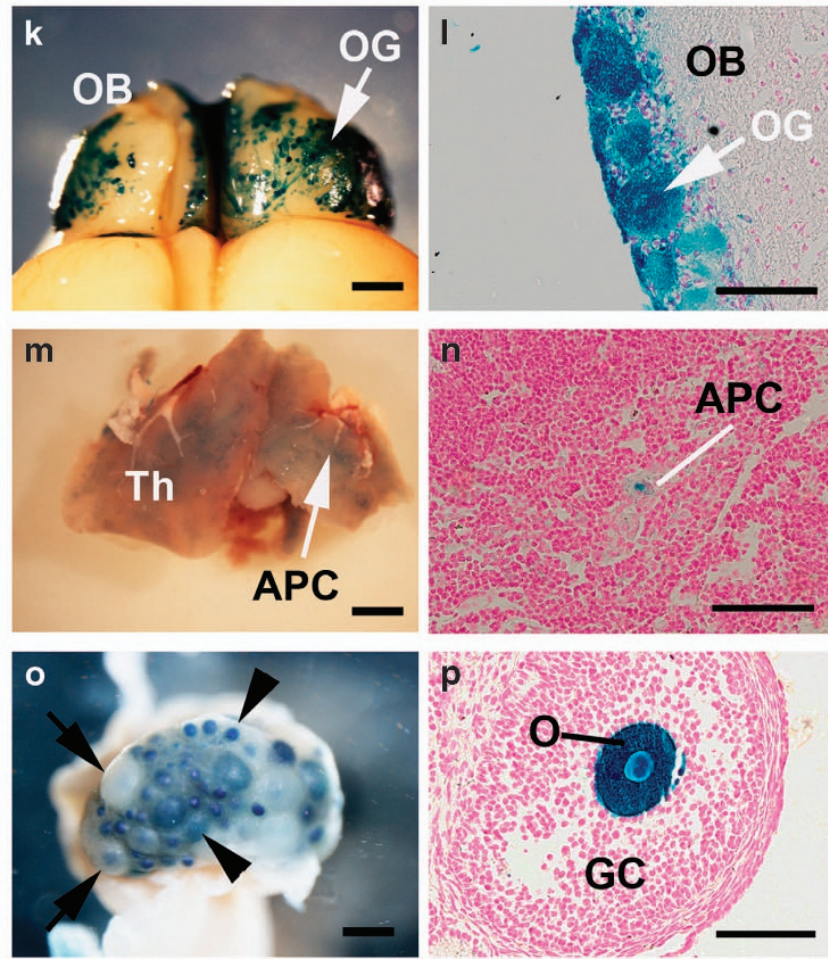

Figure 3 Expression of the pW224 transgene visualized by $\beta$-galactosidase activity to confirm that the resulting transgene is able to faithfully recapitulate the expression of endogenous Umodl1. Panels in the first and third columns show the lacZ positive whole mount organs; while the expression of the transgene is detailed in sections shown in panels listed in the second and fourth columns. Developmental stages of the specimens are indicated on top of the columns. Umodl1-Tg is one of the earliest developmental genes expressed in vomeronasal organ ( $\mathbf{a}$ and $\mathbf{b}$ ), thymus ( $\mathbf{a}, \mathbf{e}$ and $\mathbf{f})$, inner ear (c and $\mathbf{d})$, and embryonic ovary $(\mathbf{g}$ and $\mathbf{h})$. In the young adult mice, Umodl1-Tg expression in olfactory neurons, vomeronasal organs is maintained (i and $\mathbf{j})$. Meanwhile, axonal cells in the glomerular layer of olfactory bulbs show a high level of Umodl1-Tg (k and $\mathbf{I})$. In thymus, its expression is restricted to the antigen-presenting cells $(\mathbf{m}$ and $\mathbf{n}$ ). In the young female reproductive system, Umodl-Tg is solely expressed in the maturing oocytes (o and $\mathbf{p}$ ), which is consistent with the ISH data on the endogenous Umodl1. Arrows and arrowheads in o indicate expanded antral follicles and densely packed preantral follicles, respectively. Scale bars, $100 \mu \mathrm{m}$

higher than that of the WT controls. Upon eCG stimulation, Umodl1 is dramatically up-regulated by 3.5 - and 6.6 -fold in the WT and Tg oocytes, respectively, as compared with that in the untreated WT ovaries (Figures $4 e$ and f). In the absence of exogenous eCG, neither WT nor Tg cumulus cells at this age are Umodl1 positive. Nevertheless, in vivo injection of eCG turned Umodl1 on in the WT cumulus cells. Furthermore, the extra copies of the transgene rendered another1.7-fold increase in Umodl1 production (Figures $4 \mathrm{~g}$ and h). In conclusion, Umodl1 is a gonadotropin-regulated gene, which shows prompt and robust responses to the hormonal stimuli.

Multiple reproductive defects in the ageing $\mathrm{Tg}$ females. Reproductive parameters, including onset of puberty, fertility, litter size and ovarian follicular reserves were compared between $p W 224 \mathrm{Tg}$ and healthy control females. The control mice were from a Lycat/lacZ Tg line, pW209, in which no abnormality in fertility has ever been observed. ${ }^{17}$ All pW224 Tg mice entered puberty and exhibited mating behavior. The first copulatory plugs were detected around 2 months of age for both experimental groups. Approximately $78 \%$ of the vaginally plugged $p W 224$ females delivered pups, and the litter size was not different from the controls. However, pW224 females plugged at 3 months of age produced fewer pups than the controls. Catastrophic decline in fertility was observed between 3 and 4 months of age. Only $30 \%$ of the 4 month-old Tg mice were able to deliver pups, and the litter size was far smaller than the controls (control, $9.8 \pm 1.3$ versus pW224, $2.3 \pm 0.2 ; P<0.01$ ). No Tg females have ever produced more than three litters. All Tg females older than 6 months became anovulatory (Figure 5a). When the total 

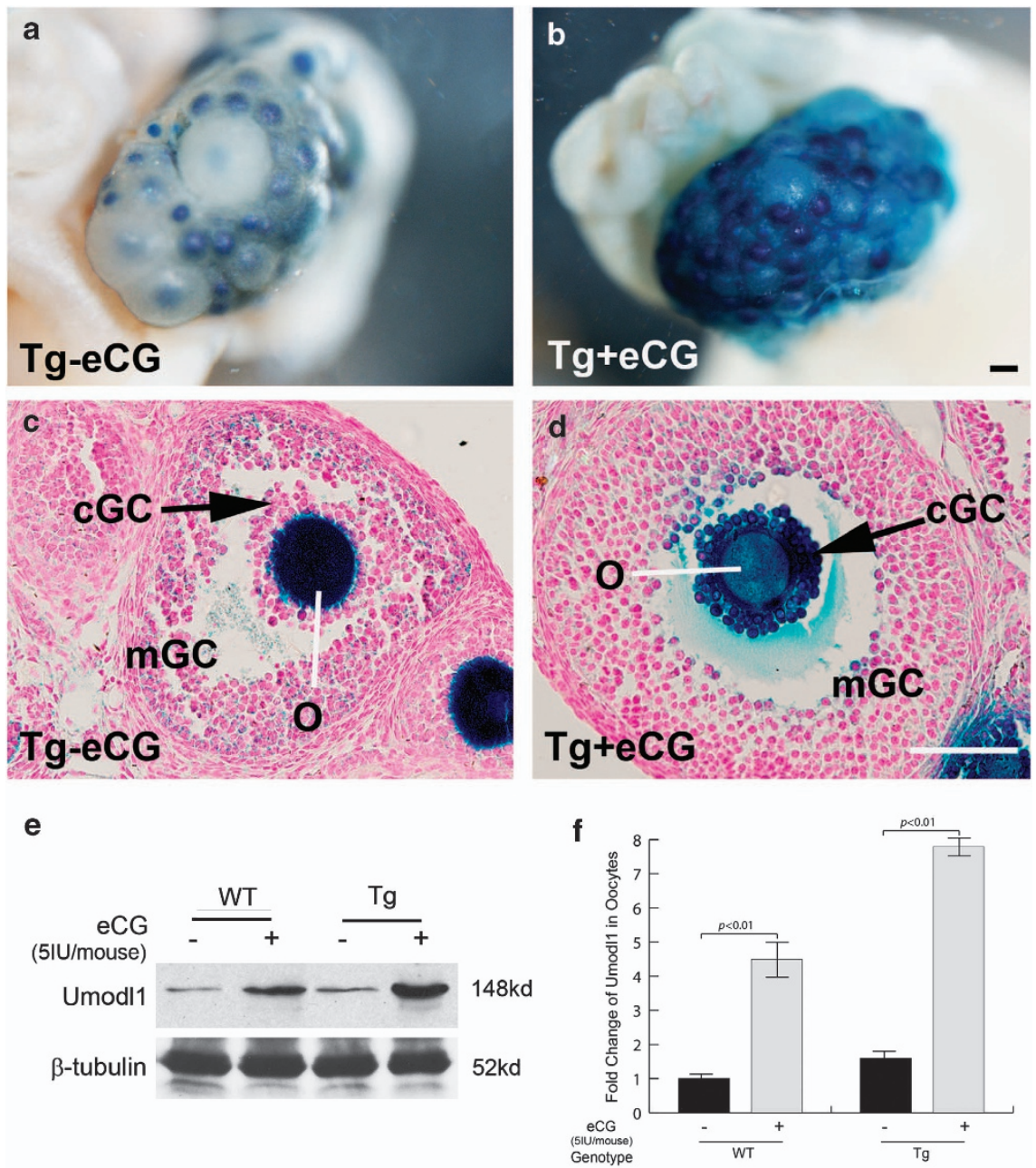

g

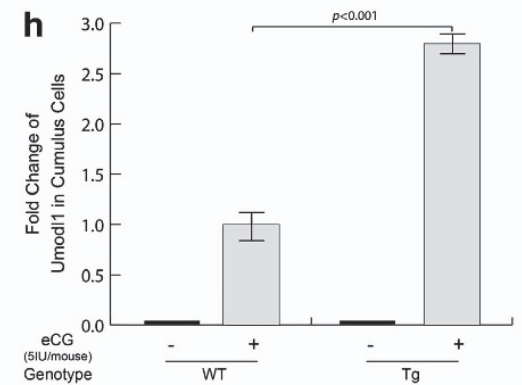

Figure 4 Stimulation of pW224 transgene by gonadotropin. Three-week old Tg control (Tg-eCG) and eCG-stimulated (Tg + eCG; $5 \mathrm{IU}$ per mouse) Tg ovaries were stained for $\beta$-galactosidase expression as wholemounts ( $\mathbf{a}$ and $\mathbf{b}$ ) and then serial sectioned (c and $\mathbf{d}$ ). Ectopic expression of Umodl1-BAC is observed in the cGCs of the stimulated ovarian follicles (d). Scale bars in sections represent $50 \mu \mathrm{m}$. Regulation of Umodl1 by gonadotropin was tested in vivo by western blot assay on WT and Tg oocytes (e) and cumulus cells $(\mathbf{g})$, and quantified by NIH ImageJ ( $\mathbf{f}$ and $\mathbf{h}$, respectively).Ovarian cells from untreated mice were included as controls. $\beta$-tubulin was used as sample loading control

number of ovarian follicles under maturation was counted, a rapid deterioration of follicle reserve (approximately a $78 \%$ decline) was evident in the 4-month-old Tg mice (Figure 5b). Among 6-month-old Tg ovaries, no oocytes were morphologically distinguishable (Figures $5 \mathrm{f}$, $\mathrm{i}$ and $\mathrm{o}$ ). When serum levels of $\mathrm{FSH} \beta$ were examined, significant increases were observed from 3 months of age in the $\mathrm{Tg}$ females and maintained at a high level even after the ovaries degenerated
( $\geqslant 6$ months). Elevated FSH $\beta$ in the Tg mice demonstrates classic signs of hypogonadotropic hypogonadism, typical of premature ovarian failure (Figure 5c; Supplementary Figure $3 a) .{ }^{3}$ Early gonadal dysfunction was further confirmed by the expedited decline of AMH levels in the Tg sera (Figure $5 \mathrm{c}$; Supplementary Figures $2 c-d$ and $3 b$ ), in agreement with the morphometric analysis that showed a rapid diminution of the maturing ovarian follicle pool in the Tg ovaries (Figure 5b). 
Normally, the ovarian cortex consists of ovarian follicles embedded in the stromal cells. At 4 months of age, a noticeable number of $C L$ can be seen in the periphery of ovary (Figure $5 \mathrm{~d}$ ). Morphologies of healthy preantral and antral follicles are shown in Figures $5 \mathrm{j}$ and $\mathrm{m}$, as well as Supplementary Figure 2a. However, compared with the controls, the 4-month-old $\mathrm{Tg}$ ovaries contained very few follicles, and were devoid of any distinguishable CL (Figures $5 e, h$ and $n$ ). In addition, an extensive network of blood vessels (Bv) invading the cortex was prominent (Figure 5h; arrows, Figure $5 \mathrm{n}$ ); and a significant number of mutant follicles were filled or surrounded presumably by fatty tissue (arrow, Figure 5h). The majority, if not all, of the Tg oocytes were either fragmented or deformed (Figure 5k). Strikingly, all of the somatic cells in the mutant ovaries displayed a pycnotic appearance, indicative of increased apoptotic activities. The mutant GCs were disorganized, and tended to dissociate from each other and from the theca layer, suggesting a defective cell-cell interaction among them (Figure 5k; Supplementary Figure $2 b$ ). In the Tg follicles, the GCs immediately adjacent to oocytes were lacZ positive, reminiscent of cumulus cells in the young $\mathrm{Tg}$ follicles after eCG stimulation (Figure 4d; Supplementary Figure 2b). Histology failed to detect any primordial follicles underneath the germinal epithelium, indicating a complete depletion of the primordial reserve in the 4-month-old mutant ovaries (arrowheads, Figure 5n; Supplementary Figures $1 \mathrm{f}-\mathrm{h}$ ). No gross abnormalities were detected in uterus at this stage (Figures $5 p$ and $q$ ). At 6 months of age, the mutant ovaries shrank to one-twentieth the size of the control ovary and were devoid of any 'normallooking' follicles (arrows, Figure 5i and I). Dislocated ovarian bursas were seen in some Tg ovaries (asterisks, Figures $5 f$ and i). Accumulation of lipid droplets was observed in all of the 'follicle-like' compartments (arrows, Figure 5i). The germinal epithelial layer was missing (arrowheads, Figures $5 \mathrm{l}$ and o). Pyknotic dead nuclei were primarily found in the cortex; whereas the cells in the medulla adopted an irregular morphology and became nuclear-fast-red negative, suggesting that the nuclear structures were destructed and the cells had lysed already (asterisks, Figures $5 \mathrm{I}$ and o). Secondary to the ovarian defects, particularly the absence of CL at 6 months of age, the anatomy of the Tg uterus was severely affected, characterized by condensed pyknotic nuclei in all uterine cells and the detachment of glandular epithelial linings from the supporting stromal cells, resembling uterine atrophy phenotype typically found in ovariectomized mice (arrows, Figure 5r). The histological data point to earlier apoptotic events followed by necrotic cell death in the $\mathrm{Tg}$ ovaries between 4 and 6 months of age. Apoptosis was evaluated in situ by TUNEL. No TUNEL signal was detected in the control secondary follicle (Figure 6a). However, when morphologically comparable follicles from the $\mathrm{Tg}$ mice were tested, elevated apoptosis in the nucleoli of the oocyte and the surrounding GCs was evident (arrows and arrowheads, respectively; Figure $6 \mathrm{~b}$,). In the 6-month-old ovary, although the overall cellular architecture of the follicle was still discernible, the majority of the cells were DAPI-negative. Almost all of the remaining DAPI-stained cells were TUNEL positive (Figure 6c). Genomic DNAs from WT and degenerating $\mathrm{Tg}$ ovaries were also electrophoresed for DNA ladder detection. There was no difference in the DNA migration patterns between WT and 2-month-old Tg ovaries. In contrast, a significant amount of genomic DNA isolated from 4-monthold Tg ovaries exhibited the 'ladder' pattern characteristic of apoptosis. Furthermore, the low concentration and 'smear' migration pattern of DNA from 6-month-old $\mathrm{Tg}$ ovaries indicated that necrosis was predominately responsible for the ovarian degeneration at this stage (Figure $6 \mathrm{~d}$ ).

Early cyst formation was observed in the Tg ovaries from 4 months of age. Almost all of the large follicles were 'empty' cysts with a few GCs clustered in the lumen (Figure 6e). Oilred-O staining further confirmed that these follicles were actually filled with lipid droplets (Figures $6 f$ and g). Abnormal fat deposition in a particular tissue usually suggests a degenerative change, and is considered as the cause of loss of function or a cause of death. Q-PCR analysis on peroxisome proliferator-activated receptor gamma (PPAR $\gamma)$ confirmed an increased level of adipogenesis in the 4-month-old Tg ovaries. No PPAR y expression was detected from the 6-month-old Tg ovaries due to the availability of viable cells (Figure 6h).

\section{Aberrant Umodl1 expression and disturbed molecular properties of the $\mathrm{Tg}$ ovarian cells. The correlation} between accelerated ovarian ageing and the age-related aberrant expression of Umodl1 was tested by Q-PCR and western blot assays. As expected, similar dynamic patterns of Umodl1 expression were observed at both mRNA and protein levels during ageing development of the $\mathrm{Tg}$ mice (Figures 7a and b; Supplementary Figures 3c-e). Briefly, unlike the WT GCs, the Tg cumulus GCs showed ectopic expression of Umodl1 when examined at 2 months of age. Its expression reached a peak level around 3 months of age, coinciding with the surge of circulating $\mathrm{FSH}$ in the $\mathrm{Tg}$ females (Supplementary Figures $3 a$ and c). By 6 months, it became undetectable. At all ageing time points tested, Umodl1 levels in WT ovarian tissues are generally constant; whereas the Tg oocytes and whole ovaries exhibited notably higher levels of Umodl1 upto 3 months of age, followed by an accelerated pace of decline in Umodl1 expression. In addition, BCL-2associated $X$ protein (Bax), a pro-apoptotic bcl-2 family member that is linked to physiologic apoptosis, was consistently elevated in the $\mathrm{Tg}$ ovaries from 2 months of age (Figure 7b; Supplementary Figure 3f). Therefore, the dynamic changes of Umodl1 in the $\mathrm{Tg}$ mice is closely correlated with the progression of the premature ovarian ageing.

The molecular defects underlying the mutant phenotype was further investigated using mRNA in situ hybridization (ISH). Several ovary-specific genes were tested on paraffin sections bearing comparable morphologies of follicles from 4month-old WT and Tg ovaries (Figure 7c). Gdf-9 is an oocytespecific gene that mediates crosstalk between oocytes and GCs. At early follicular stage, Gdf-9 promotes the transition of follicles from primary to secondary stage. In pre-ovulatory follicles, Gdf-9, in conjunction with LH and BMP-15, initiate ovulation of a mature cumulus-oocyte complex. ${ }^{6,18}$ However, in the 4-month-old Tg ovaries, Gdf-9 declines to a undetectable level. Ring finger protein 35 (Rnf35) is one of the earliest oocyte-specific genes, whose expression persists upto the blastocyst stage. Overexpression of Umodl1 inactivates 

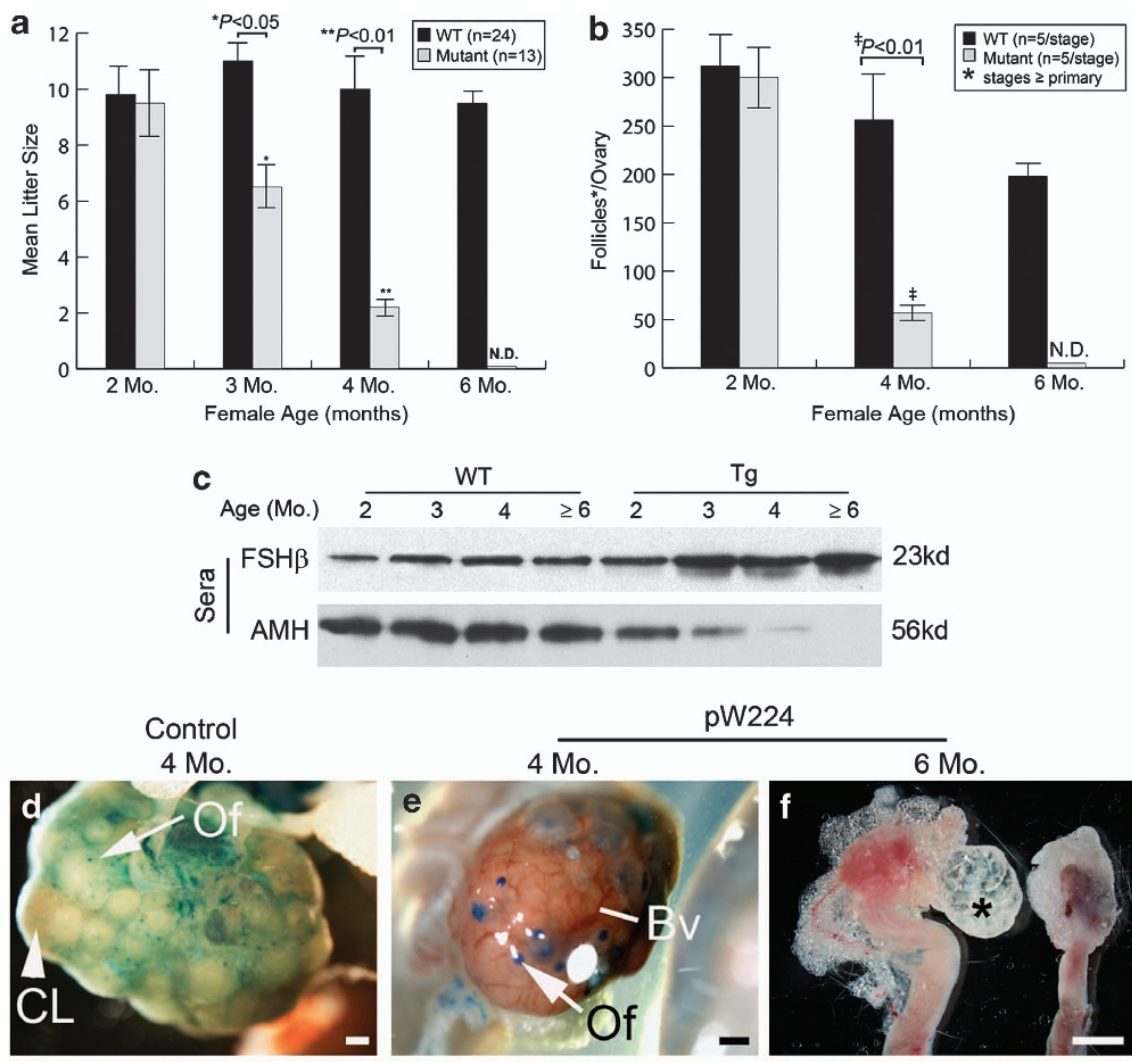

W224
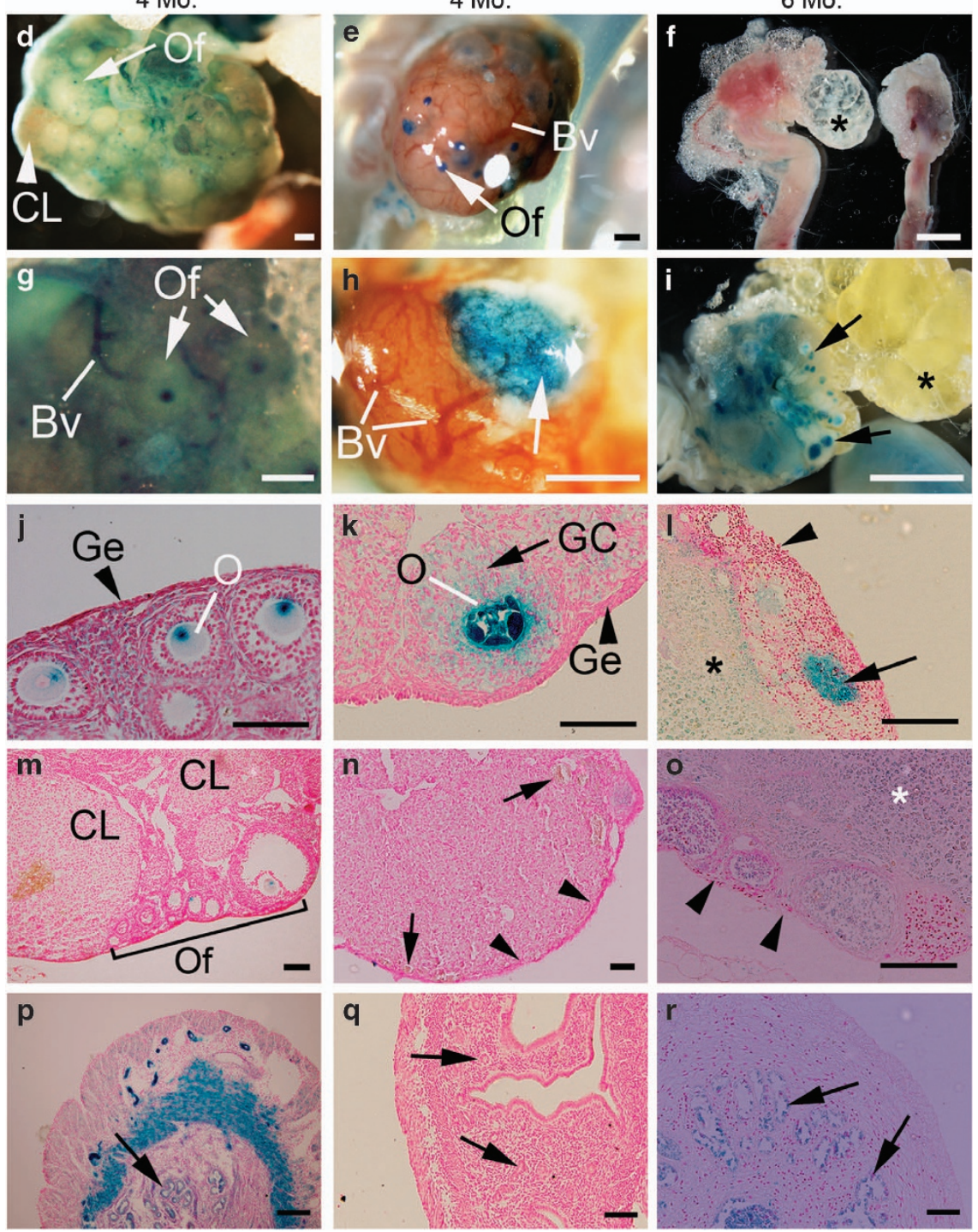
Rnf35. Granulosa cell high-mobility group-box protein-1 $(G c x-1)$ encodes a transcription regulator that is specifically expressed in the pituitary and GCs of ovary. Absence of Gcx1indicates that the molecular signature of the Tg GCs has been altered as a result of 'overdosed' Umodl1. In WT ovaries, spermatogenesis- and oogenesis-specific basic helix-loophelix1 $(\mathrm{NOHLH})$ is moderately expressed in the CL; whereas its expression was drastically up-regulated in the $\mathrm{Tg}$ ovarian cortex. In contrast, the anti-apoptotic gene $\mathrm{Bcl}-2$ like-10 $(B c / 2 / 10)$ remains unchanged in the mutant oocyte, suggesting a self-protective mechanism adopted by the degenerating oocytes. These ISH data, in combination with the dynamic expression of PPAR (Figure 6h), clearly indicate that the molecular signature of the Tg ovarian cells have already been changed at 4 months of age. Consequently, mice overexpressing Umodl1 are doomed to early follicular depletion and premature ovarian failure.

\section{Discussion}

Mice carrying extra Umodl1 alleles delivered normal litter sizes at young age, and then beyond 4 months, litter size decreased rapidly culminating in premature infertility at 6 months of age. Overactive Umodl1 signaling imposed adverse effects initially on the wellness of oocytes themselves, followed by mis-communication between oocytes and GCs. Mis-instructed GCs were more prone to apoptosis or adopting an adipogenic fate. Consequently, as early as 4 months of age, the ovaries lacked well-defined structure typical of CL, and displayed a complete loss of primordial follicles. The few remaining oocyte, enclosed by deranged GCs, were either fragmented or deformed. By 6 months of age, ovarian function failed completely. This study undoubtedly provides another line of evidence to define mechanisms of reproductive function, as well as a putative target for infertility therapy. One may come up with the question: why the overdosed Umodl1 exerted its detrimental effects on ovaries around 4 months of age, instead of even younger age? This may be explained by the stimulatory interaction between Umodl1 and FSH. Our expression data have demonstrated that Umodl1 is positively regulated by $\mathrm{FSH}$ in ovary. It has long been confirmed that circulating $\mathrm{FSH}$ increases gradually with age in both humans and mice. $2,19-21$ Additionally, lack of proper feed-back inhibitory signals from our 'defective' Tg ovaries to the hypothalamus-pituitary results in elevated $\mathrm{FSH}$, which may in turn adversely augment the Umodl1-mediated signals. As this stimulatory loop advances, a catastrophic ovarian degeneration becomes inevitable (Figure 8).

Preferential expression of Umodl1 suggests its potential roles in coordinating the functions of neuroendocrine, immune and reproductive systems. Umodl1 is one of the earliest genes expressing in the olfactory epithelium and VNO neurons in mice. Later, its transcripts extend to the olfactory and $\mathrm{GnRH}$ neurons (Supplementary Figures 1c-e). Involvement of VNO in reproduction and social behavior has been reported in many species. ${ }^{22-24}$ Ablation of VNO-mediated pheromone signaling in mice eliminates the acceleration of puberty, the suppression of estrus cyclicity and pregnancy block to strange males. ${ }^{22,25,26}$ A thymus-pituitary-ovarian axis has been proposed in controlling female fecundity. Congenital athymia or neonatal thymectomy in mice leads to ovarian dysgenesis, premature ovarian failure, and ovarian neoplasia. ${ }^{27,28}$ Female patients with thymic aplasia also have dysgenetic ovaries. ${ }^{29}$ Reciprocally, ablation of ovarian function by ovariectomy results in thymus enlargement due to the cession of estrogen production. ${ }^{30,31}$ Moreover, absence of ovarian hormones reprogramed the $\mathrm{T}$-cell repertoire, leading to an increased diversity of T-cell sub-populations. Even though it is clear that thymus disturbances hasten ovarian ageing, until today, the molecular mechanisms linking thymus and gonads are elusive. Umodl1 is a candidate gene that shows an abundant expression in both ovaries and thymic/peripheral T cells upon induction. Our preliminary data on 4-month-old Tg thymuses do show that, aside from a drastic reduction in size and weight, their cellularity is severely compromised (Supplementary Figure 4).

$\mathrm{Tg}$ animal models help us greatly to dissect the genetic components responsible for different forms of reproductive dysfunction. Oocyte-specific overexpression of BMP-15 skewed the follicle pool towards secondary follicles. In the adult mice, an increased number of atretic follicles led to an expedited decline in the ovarian reserve. ${ }^{32}$ Female mice lacking estrogen receptor $\alpha$ produced increased levels of luteinizing hormone receptor in the GCs. Consequently, the maturing follicles arrested at pre-ovulatory stage and destined to atresia. ${ }^{33}$ Similarly, inactivation of forkhead box L1 led to early initiation of folliculogenesis among the primordial follicles, thus resulting in uncoupled proliferation/differentiation of GCs and follicular atresia. ${ }^{34}$ Compared with the

Figure 5 Multiple reproductive defects in the Umodl1-Tg females. Accelerated decline in litter size (a) and number of maturing follicles per ovary (b) were observed in the $\mathrm{Tg}$ females. Error bars indicate S.D. Litter size and follicle number between WT and Tg mice at specified age were compared using Student's t-test. Genotypes and sample size are indicated in the upper right corners of panels $\mathbf{a}$ and b. N.D., not detectable. (c) Western blot assay to examine the dynamics of serum FSH $\beta$ and AMH levels as the WT and Tg females aged. Genotype and sample age are indicated on the top. (d-r) Histological analysis showing the progression of ovarian degeneration. A 4-month-old LycatBAC Tg ovary was included as the healthy control (panels in the left column). (d-i) whole mount views of freshly isolated (f) or lacZ-stained (d, e, $\mathbf{g}, \mathbf{h}$ and i) ovaries from the control and ageing $p W 224$ mice. Panels $\mathbf{g}$ and $\mathbf{h}$ are the higher power views of panels $\mathbf{d}$ and $\mathbf{e}$, respectively. Panel $\mathbf{i}$ shows the left ovary in panel $\mathbf{f}$ at a higher magnification after lacZ-staining. Panels $\mathbf{j}-\mathbf{o}$ are sectioned control $(\mathbf{j}$ and $\mathbf{m})$ and mutant $(\mathbf{k}, \mathbf{i}, \mathbf{n}$ and $\mathbf{~} \mathbf{0})$ ovaries. Panel $\mathbf{m}$ is a representative photograph showing all types of ovarian cells, including $\mathrm{CL}$, interstitial stromal cells, and follicles of various stages (of primordial, primary, secondary \& pre-ovulatory follicles). The arrows in panel $\mathrm{i}$ show the degenerated follicles filled with lipid droplets. The arrowheads in panels I and 0 indicate the absence of germinal epithelial layer in a 6-month-old ovary. The arrow in panel I indicates a degenerated follicle devoid of oocyte. Arrowheads in panel $\mathbf{n}$ show the absence of primordial ovarian follicles in the cortex region. Arrows in panel $\mathbf{n}$ indicate abnormal blood vessel formation in the Tg ovary. Higher power views of the regions specified by arrows and arrowheads in panel $\mathbf{n}$ can be found in Supplementary Figures $1 f-h$. Regions marked by asterisks in Panels I and $\mathbf{o}$ are the ovarian medulla that has become nuclear-fast-red negative. Secondary to the ovarian defects, progressive abnormalities can be seen in the mutant uteri $(\mathbf{p}-\mathbf{r})$. Arrows in panels $\mathbf{p}-\mathbf{r}$ specify the uterine glandular epithelial cells. Genotype and age are indicated on the top of each column. Scale bar in sections, $100 \mu \mathrm{m}$ 

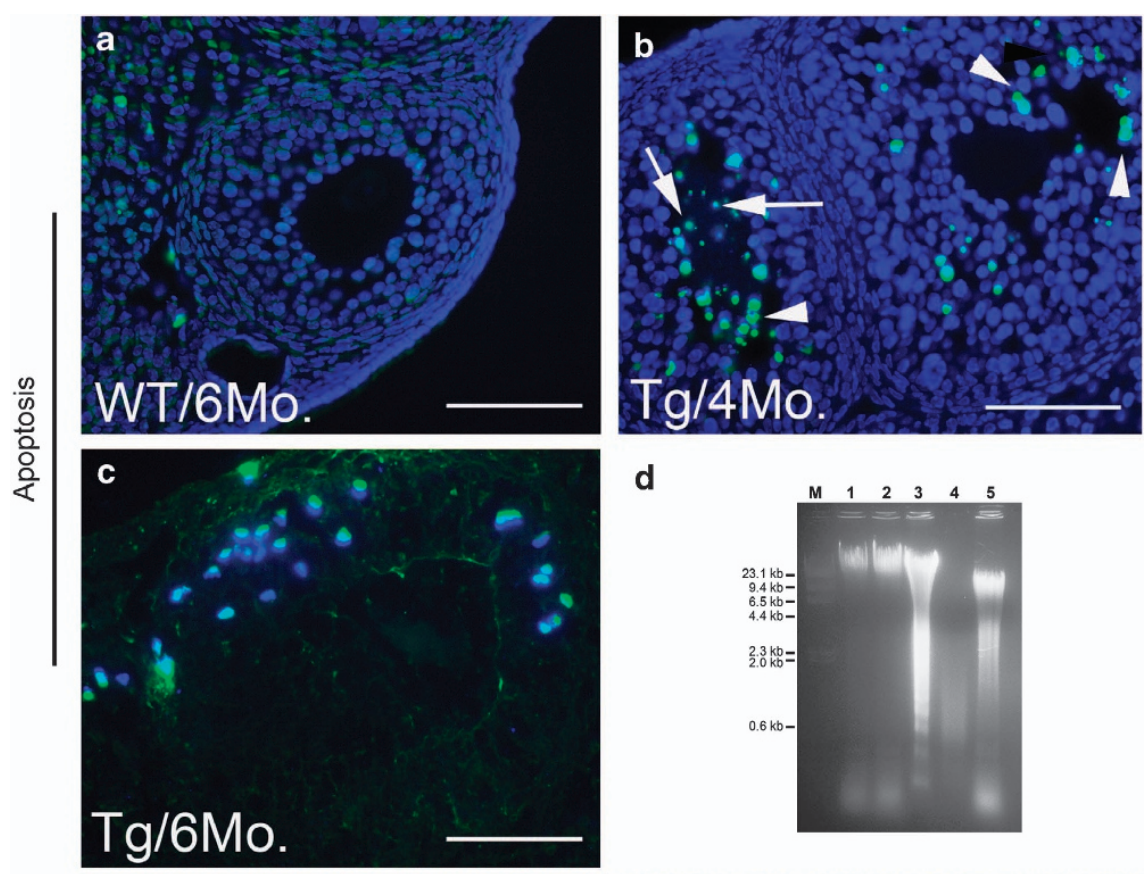

d
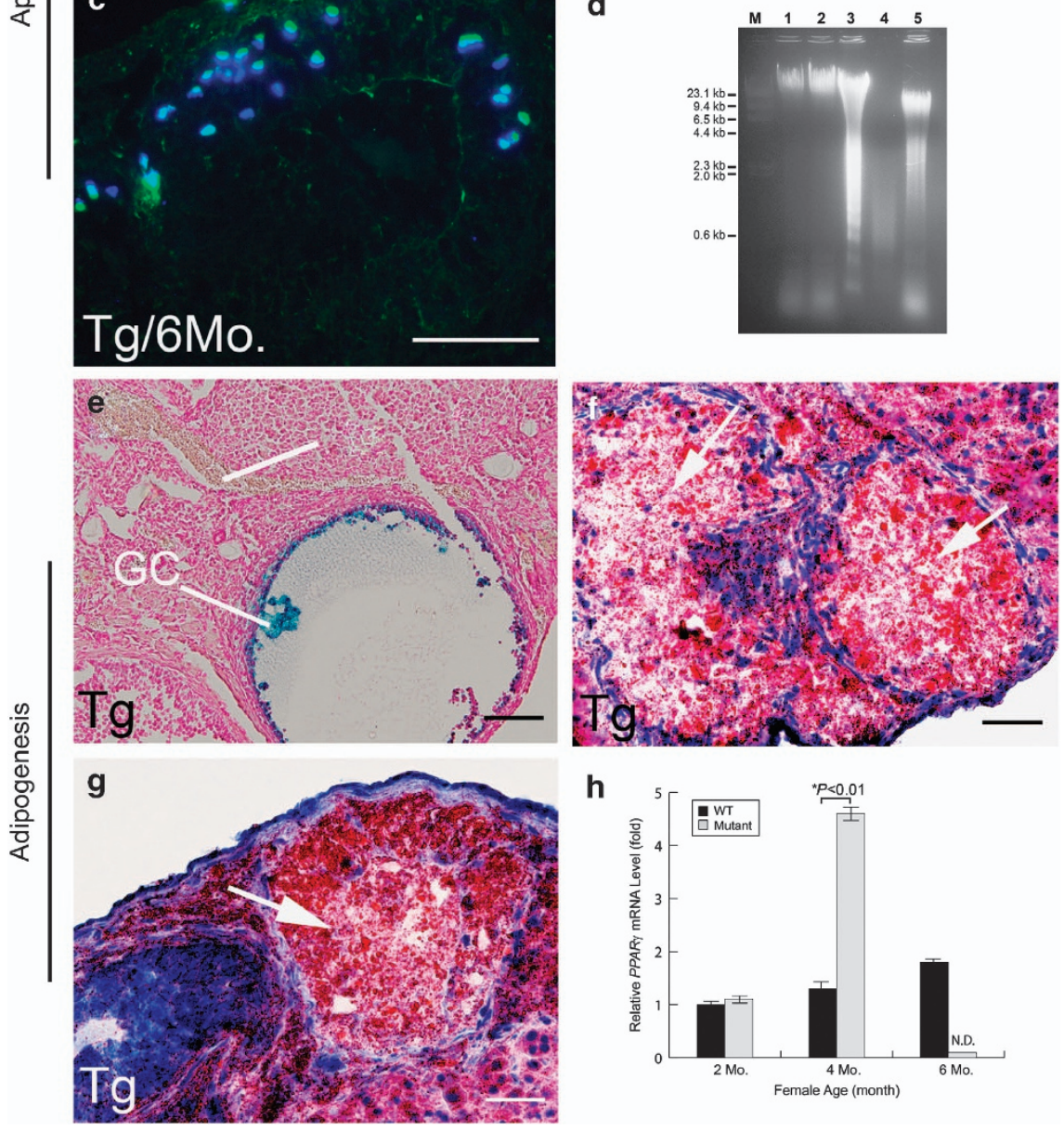

Figure 6 Elevated apoptotic activity and lipid deposition in the Tg ovarian follicles. TUNEL assay on 6-month-old WT (a), 4-month-old Tg (b) and 6-month-old Tg (c) ovaries was performed. (d) Agarose gel electrophoresis to detect DNA fragmentation using genomic DNA from 6-month-old WT (lane1), 2-month-old Tg (lane 2), 4-monthold Tg (lane 3) and 6-month-old Tg (lane 4) ovaries. A necrotic control was also included (lane 5). No difference in DNA migration pattern was observed between samples from WT and 2-month-old Tg ovaries (lanes 1 and 2). Elevated apoptosis as exhibited by the 'ladder' DNA pattern became apparent in Tg ovaries from 4 months of age (lane 3). In 6-month-old Tg ovaries, low-molecular-weight DNA fragments were present as a smear pattern, indicating that necrosis is prominent at this stage (lane 4). (e-h) Abnormal adipogenesis in 4-month-old Tg follicles. (e) is a representative lacZ-stained section showing a large cystic follicle ( $>400 \mu \mathrm{m}$ in diameter, which is equal to the size of a healthy antral follicle). Oil-Red-O staining shows these cystic follicles were essentially filled with lipid drops (arrows, $\mathbf{f}$ and $\mathbf{g}$ ). (h) Expression of adipogenic marker gene PPARy in 2,4 and 6-month-old ovaries from WT and Tg females was examined by Q-PCR. For all experiments, each sample input was normalized by GAPDH expression levels. PPARy expression was presented relative to that of the 2-month-old WT ovaries as the mean \pm S.E.M. of three independent experiments. Data were analyzed using a one-way $t$-test. Genotype and ovarian age are indicated in the lower left corners of the corresponding photographs. Scale bars, $100 \mu \mathrm{m}$

above-mentioned mutants, our Umodl1 gain-of-function mutant is unique, in which excessive Umodl1 exerts its effects initially on oocytes. Then, the defective oocytes send destructive signals to the neighboring somatic cells, further leading to disorganization and cell-fate alteration in GCs.
Unlike other mutants in which hemorrhagic or fluid-filled cysts were commonly found, the Umodl1-Tg follicles were filled with fat cells, suggestive of a progressive conversion of GCs to adipocytes. The detrimental effect of the increased Umodl1 on the immune and reproductive systems was augmented with 

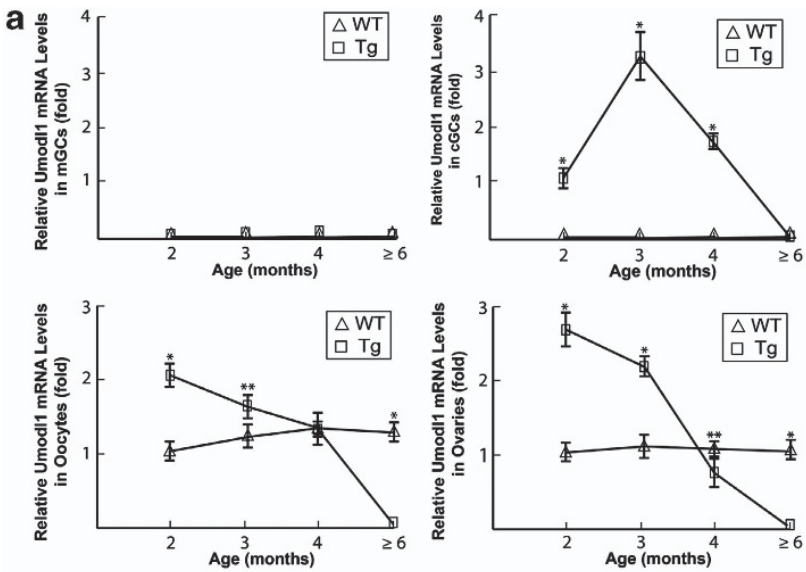

b

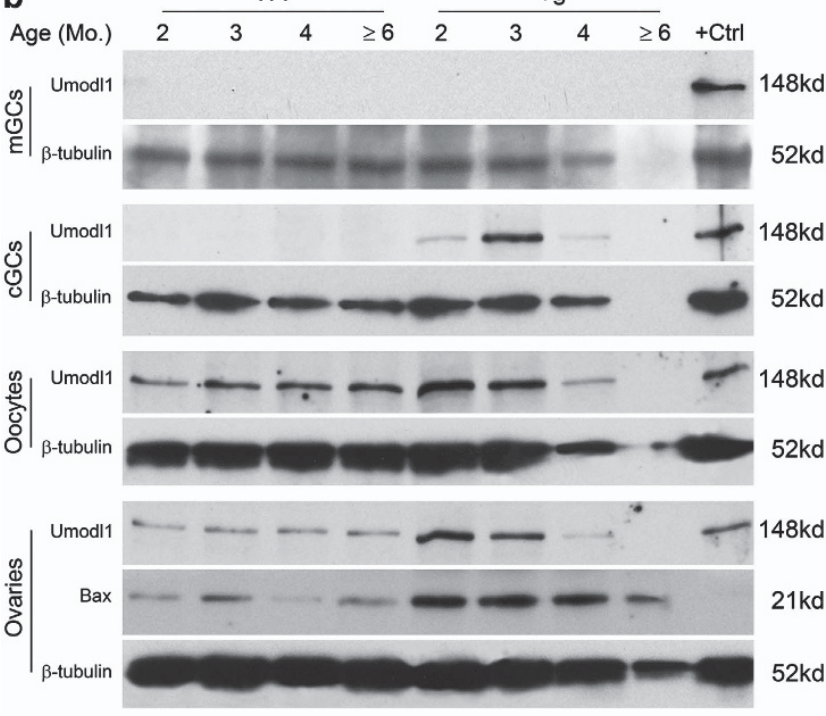

c

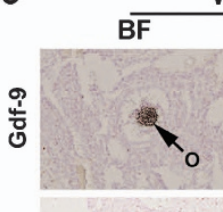

WT

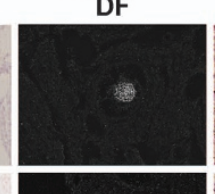

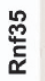
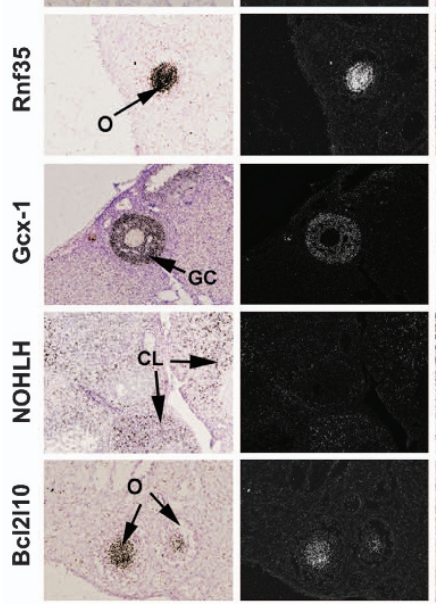

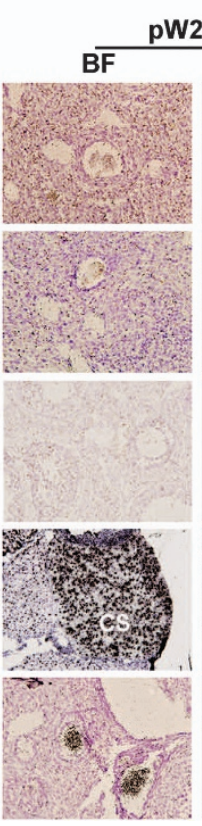

pW224

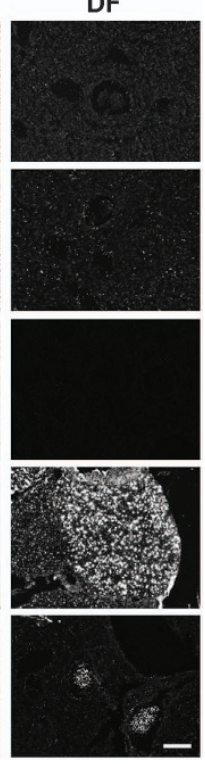

age. Accordingly, high rates of morbidity and mortality were prominent, and the majority of our Tg mice died by 8 months of age.

Taken together, we present another animal model for premature ovarian failure and accelerated ovarian aging. In-depth analysis of this mutant will expand our knowledge to decipher the underlying mechanisms leading to female infertility.

\section{Materials and Methods}

RNA in situ hybridization. The mouse Umodl1 gene was isolated in our laboratory by subtractive hybridization screening between cDNAs from untreated and gonadotropin-stimulated mouse ovaries (data not shown). Preferential display of Umodl1 was examined in this study by RNA ISH, Northern blot assay and immunofluorescence labeling methods.

Thymuses from 28-day old WT mice and ovaries from 4-month-old WT and pW224 Tg mice were collected. Specimen treatment, hybridization, washing and autography were essentially performed as described previously. ${ }^{17,35}$ Ovarian sections were hybridized with ${ }^{35} \mathrm{~S}$-labeled anti-sense RNA probes. Developed sections were finally counter-stained with haematoxylin. Paraffin sections of WT thymuses were hybridized with the digoxigenin-labeled Umodl1 RNA probe. Sample preparation, hybridization and signal detection were carried out as previously described. ${ }^{17}$ Information on plasmids for preparing riboprobes for Umodl1, Gdf-9, Rnf35, GcX-1, NOHLH and Bcl2/10 is available upon request.

Northern blot analysis. Female mice of 21-day old were injected with eCG at $5 \mathrm{IU}$ per mouse. Ovaries were collected at different time points from $0-72 \mathrm{~h}$ after injection. Neonatal ovaries were included as a negative control. RNA extraction, blotting and signal detection were performed as previously described. ${ }^{36}$ Approximately $5 \mu \mathrm{g}$ of total RNA was loaded in each lane. The ${ }^{32} \mathrm{P}$-labeled Umodl1 probe was synthesized from the purified coding region of mouse Umodl1 by the random hexamer labeling method.

Transgene construction and Tg mice generation. The Umodl1 BAC clone, RP24-430m9 was purchased from the Children's Hospital Oakland Research Institute. To generate the targeting vector, a 455-bp genomic fragment located at the $3^{\prime}$-UTR of Umodl1 was PCR-amplified using Oligo 511 (5'-GC TAATTCATCGAACATTGACGGG-3') and Oligo 512 (5'-GACTCGTTAG AAAGCGTTC CGGTT-3'), and subcloned into $\operatorname{pBSSKII}(-)$, generating plasmid

Figure 7 Spatial and temporal profile of Umodl1 expression in ovarian follicles of aging $\mathrm{Tg}$ mice and disturbed ovary-specific gene regulatory pathways in response to Umodl1 overexpression in the 4-month-old Tg mice. (a) SYBR green-based quantitative PCR assay was employed to determine the relative levels of Umodl1 in mGCs, cGCs, oocytes and whole ovaries of WT and Tg female mice $(n \geqslant 3$ per genotype) at various time points. Quantity of each sample was normalized to expression of GAPDH $(\mathrm{Ct}=14.8-15.6)$. No significant levels of Umodl1 were detected in the WT mGCs and cGCs, as well as the Tg mGCs (Ct>25). Umodl1 levels in Tg cGCs of different ages were compared with that of 2-month-old $\mathrm{Tg}$ cGCs; while levels of Umodl1 in oocytes and ovaries were compared with those from the 2-month-old WT samples, respectively. Data points represent mean \pm S.E.M. of three repeats. ${ }^{*}$ and ${ }^{* *}$ represent significant difference $P<0.01$ and $P<0.05$, respectively, as compared with the age-matched WT controls. (b) Representative western blots showing expression of Umodl1 in different ovarian cells at various ages before a complete degeneration of the $\mathrm{Tg}$ ovaries. Samples from age-matched WT cells per tissues were included. $\beta$-tubulin was used as the internal control. Tissue lysate from a 2-month-old WT spleen was used as the positive control for Umodl1 $(+\mathrm{Ctrl})$. The blot loaded with lysate from whole ovaries was stripped and re-probed with a Bax antibody to demonstrate an elevated apoptosis in the Tg ovaries. (c) Altered expression patterns of key ovary-specific genes in the ovaries of pW224 Tg mice at 4 months of age. In situ hybridization using ${ }^{35}$ S-UTP labeled antisense probes was performed. Panels in columns 1 and 3 are the bright field views of tissue sections, while panels in columns 2 and 4 are photographs showing corresponding dark field views to visualize the hybridization signals. Umodl1 genotype is indicated on the top. The molecular markers examined are listed on the left. Scale bar, $100 \mu \mathrm{m}$ 

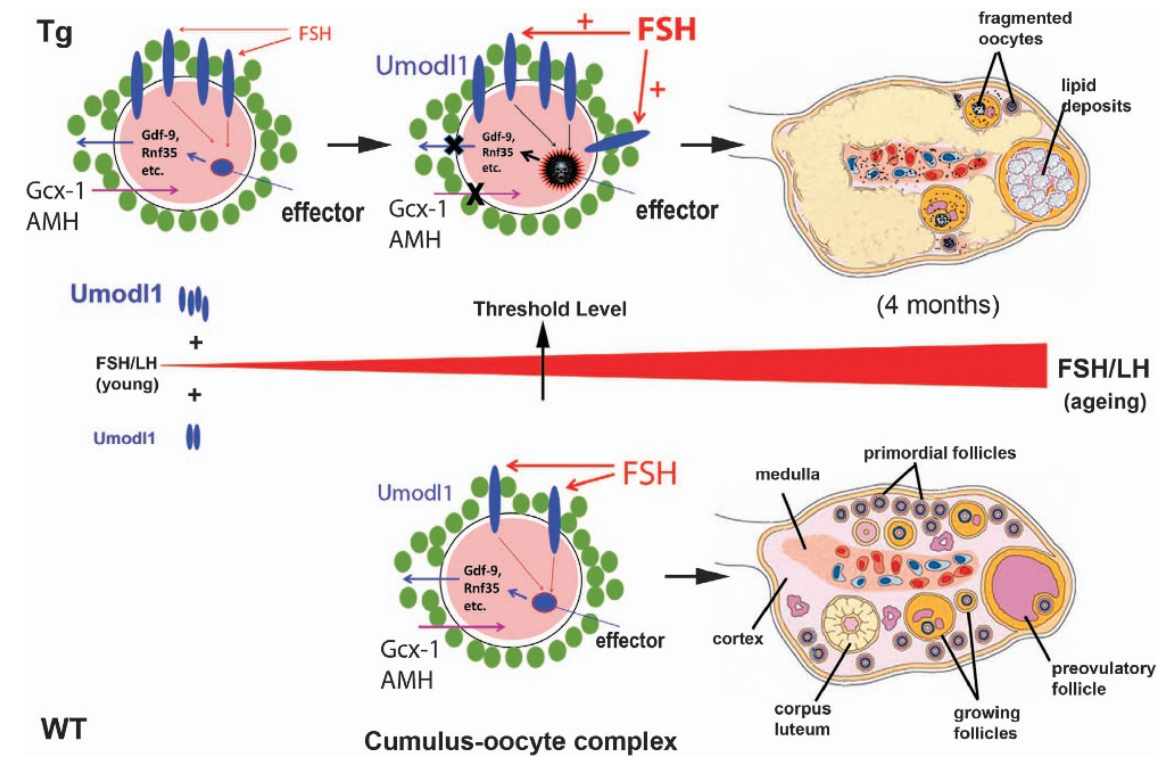

Figure 8 Hypothetic model for Umodl1 function on ovarian ageing. This schematic diagram summarizes the reproductive defects caused by Umodl1 overexpression. Meanwhile, a putative stimulatory interplay of $\mathrm{FSH} / \mathrm{LH}$ and Umodl1 on accelerating ovarian ageing is proposed based on the close correlation between the ovarian defects with the abnormal levels of circulating FSH in the mutants. At early reproductive age, the 'defective' ovaries intrinsic to Umodl1-overexpression send faulty signals to the hypothalamus-pituitary, thus resulting in elevated circulating FSH. Once the plasma FSH/LH reach the threshold levels as the ovarian deterioration proceeds, FSH/LH may act directly or indirectly on the over-expressed Umodl1. Consequently, the signaling pathway(s) mediated by Umodl1 is further amplified, imposing detrimental influences on the health of oocytes themselves. Fragmented oocytes fail to correctly instruct the proliferation and differentiation of GCs, resulting in increased apoptosis and cell-fate alteration. Collectively, these adverse impacts lead to the eventual collapse of ovarian function.

pW221. To finalize the targeting vector, the reporter cassette, ires.lacZ/GT1.2-neo was inserted into the unique BamHI site of pW221, resulting in pW223. The $5^{\prime}$ - and $3^{\prime}$-targeting arms are 317 and $138 \mathrm{bp}$, respectively. Preparation of competent Escherichia coli cells, transformation of pRedET and targeting fragment, and L-arabinose induction were performed as previously described. ${ }^{17}$ Correct targeting was confirmed by PCR amplification using Oligo 513 (5'-AATGCTCCATCTGCA CACATGCCTGGTGC-3') and Oligo 309 (5'-TCAACAGACCTTGCATTCCT TTGGCGAGAGG-3') for the $5^{\prime}$ insertion site, and Oligo 251 (5'-CGCCTTC TATGAAAGGTTGGGCTTCGGA-3') and Oligo 514 (5'-CAGCATCTCCCTG GAGTGTCTGGCAAAGAATC- $3^{\prime}$ ) for the $3^{\prime}$ insertion site. The integrity of the modified BAC clone was verified by comparing the digest pattern of the recombinant $B A C$ with that of the original clone (data not shown). The modified RP24-430m9 was renamed as pW224. BAC DNA was isolated by the alkaline lysis method and further digested with Notl. The resultant $154 \mathrm{~kb}$ fragment harboring entire modified Umodl1 was purified by gel electrophoresis and electroeluted as suggested, ${ }^{37}$ followed by quantification and dilution to a final concentration of $5 \mathrm{ng} / \mu \mathrm{l}$ in the injection buffer.

Injection of pW224 into one-cell FVB embryos was performed in our laboratory. WT FVB and pW224 Tg mice were housed in the animal facility of Weill Cornell Medical College. All animal procedures were performed following protocols approved by Weill Cornell Medical College Institutional Animal Care and Use Committee. To simplify data interpretation, the $\mathrm{Tg}$ animals were only bred with WT FVB mice. Genotype was verified by PCR amplification of tail-tip genomic DNA using Oligo 251 and Oligo 514. Copy numbers of the transgene was estimated by the comparative $\mathrm{C}_{T}(2 \Delta \Delta \mathrm{Ct})$ method using genomic DNA from tail-tips of $\mathrm{F} 3$ mice of all four independent lines (Tg20, 62, 69 and 74). Primer design, parameters for TaqMan PCR and data analysis were performed exactly as previously described. ${ }^{17}$

Immunofluorescence labeling assay. Cryo-sections of thymuses from 28-day old WT mice $(n=3)$ were used for dual immunofluorescence analysis. To examine the spatial distribution of TCR $\beta$ - and Umodl1-positive cells in thymus, a rabbit anti-mouse Umodl1antibody (sc-83738; Santa Cruz Biotechnology, Inc, Santa Cruz, CA, USA) and a FITC-conjugated hamster monoclonal anti-mouse TCR $\beta$ antibody (11-5961-82; eBioscience Inc., San Diego, CA, USA) were used as the primary antibodies. The secondary antibody to detect Umodl1 is a DyLight 594-goat anti-rabbit IgG (ab96885; Abcam; Cambridge, MA, USA). Localization of
Umodl1 in the APCs in non-stimulated thymus was tested using the anti-Umodl1 antibody and an APC-conjugated hamster anti-mouse CD11c monoclonal antibody (17-011-81; eBioscience) as the primary antibodies. Umodl1 signals were visualized by staining with a DyLight 488 donkey anti-rabbit IgG antibody (ab98488; Abcam). Pre-treatment of slides, hybridization, washing and signal detection were performed essentially as described. ${ }^{17}$

Immunoblotting. $\mathrm{CD}^{+}{ }^{+} \mathrm{T}$ cells were purified from suspensions of adult WT mouse spleen cells using the CD4 ${ }^{+}$T Cell Isolation Kit II (130-095-248; Miltenyi Biotec, Bergisch Gladbach, Germany) and adjusted to $1 \times 10^{6} \mathrm{cells} / \mathrm{ml}$ in RPMIbased complete medium. TCR $\beta^{+}$thymocytes were isolated from WT thymuses (pooled, $n=3$ ) by first staining with a FITC-conjugated hamster monoclonal antimouse TCR $\beta$ antibody (11-5961-82; eBioscience), followed by magnetic cell sorting using anti-FITC MicroBeads (130-048-701. Miltenyi Biotec). Positively selected TCR $\beta^{+}$cell fraction was collected. Approximately $2 \times 10^{6} \mathrm{CD}^{+}$splenic $\mathrm{T}$ cells and $1 \times 10^{6} \mathrm{TCR}^{+}$thymocytes were plated into each well of a 24 -well tissue culture plate and cultured for upto $72 \mathrm{~h}$ in the absence (control), or presence of soluble anti-CD3 (2 $\mu \mathrm{g} / \mathrm{ml}$; ab5690; Abcam) and anti-CD28 (2 $\mu \mathrm{g} / \mathrm{ml} ;$ sc-70609; Santa Cruz) antibodies. Cells were washed with cold PBS and lysed for $15 \mathrm{~min}$ on ice in $0.5 \mathrm{ml}$ of lysis buffer containing protease inhibitors. Cell lysates $(\sim 20-30 \mu \mathrm{g}$ protein) were subjected to $10 \%$ SDS-PAGE, followed by immunoblotting.

To quantify the effect of gonadotropin on Umodl1 expression in vivo, 3-week old WT $(n=5)$ and $\mathrm{Tg}(n=5)$ were injected with eCG at 5 IU per mouse. Cumulusoocyte complexes were collected by puncturing the enlarged follicles, and then treated with hyaluronidase to separate cumulus cells from oocytes. Samples of $\sim 1 \times 10^{4}$ cumulus cells or 50 oocytes were lysed, and approximately $2.0-3.0 \mu \mathrm{g}$ of protein was loaded per sample for western blot analysis. Age-matched ovarian tissues from untreated WT and Tg mice were used as the negative controls. Relative levels of Umodl1 expression were analyzed using the public domain NIH Image program, NIH ImageJ (developed at the US National Institutes of Health and available on the Internet at http://rsb.info.nih.gov/nih-image/).

To examine age-related changes of circulating FSH and AMH in the WT and Tg mice, blood samples were drawn from tail veins of individually housed mice aged 2 , 3,4 and $\geqslant 6$ months. At least five WT or five Tg mice in estrus phase were recruited in each specified age group for this study. Stages of estrous cycle were assessed as previously described, ${ }^{38}$ using Diff-Quick stain kit (B41321/s; Adwin Scientific, 
Woodland Hills, CA, USA). Equal volumes of sera from mice of the same genotype and age were pooled to average fluctuation of FSH in each mouse. Three microliters of each pooled serum sample were taken for electrophoresis and immunoblotting. Denature of serum samples was performed in RIPA buffer at $65^{\circ} \mathrm{C}$ for $15 \mathrm{~min}$.

Isolation of mural GCs (mGCs), cumulus GCs (cGCs), oocytes and ovaries were performed essentially as previously described. ${ }^{39}$ As almost all of the Tg ovaries older than 6 months have already degenerated, no mGCs, cGCs or oocytes were able to be retrieved. Therefore, only the degenerated whole ovarian tissues from five Tg mice older than 6 months were included in this study. Additionally, due to the scantiness of mGCs and cGCs in the 4-month-old Tg ovaries, only $2 \times 10^{4} \mathrm{mGCs}$ and $1 \times 10^{4}$ cGCs $(\sim 15 \mu \mathrm{g}$ total) were obtained at this age point and lysed for western blot assay. Number of cells used in other samples, including all WT and Tg mGCs/cGCs at earlier ages, was adjusted accordingly to ensure equal loading of samples. To examine Umodl1 expression in WT and Tg oocytes and ovaries of various stages, specimens were collected and pooled from at least three females at each specified age. Samples were quantified and approximately 3.0 (for oocyte) or $15.0 \mu \mathrm{g}$ (for others) of protein was loaded on each lane for western blot assay. The western blot containing protein lysates from whole ovaries was stripped and re-probed with anti-Bax antibody to examine the correlation of the protein levels of apoptosis-prone gene Bax with the accelerated ageing of Umodl1-Tg mice.

Primary antibodies used in this study are anti-AMH antibody (ab103233, Abcam), anti-Bax antibody (sc-493, Santa Cruz), FSH $\beta$ (sc-292422, Santa Cruz), AntiUmodl1 and anti- $\beta$-actin (ab8227; Abcam), $\beta$-tubulin (T8328; Sigma-Aldrich, St Louis, MO, USA). The secondary antibodies are goat anti-rabbit IgG HRP (sc2030, Santa Cruz) and rabbit anti-mouse IgG-HRP (sc-358914, Santa Cruz). Antibodies were diluted to working concentrations according to the manufactures' instructions. Signals were detected with Amersham ECL Prime Western Blotting Detection Reagent (RPN2232; GE healthcare, Pittsburgh, PA, USA) and exposed to X-ray films for $20 \mathrm{~s}$ to $30 \mathrm{~min}$, depending on the amount of sample loaded and the abundance of proteins of interest.

Quantitative real-time RT-PCR analysis. Olfactory bulbs ( $n=3 /$ ine Note, Umodl1 expression in olfactory bulbs is not hormonally regulated) of adult mice from each independent $\mathrm{Tg}$ line, or from age-matched WT mice were collected for total RNA extraction. Expression levels of the functional Umodl1 in Tg mice were compared with the endogenous Umodl1 of the WT mice by Q-PCR method. Dynamic expression of PPAR $\gamma$ was examined as the mutant ovaries aged. Tg ovaries ( $n=3$ per stage) were collected at 2, 4 and 6 months of age. Ovaries at the same age were pooled for Q-PCR assay. An equal number of agematched WT ovaries were included as controls. Expression of Umodl1 in different types of ovarian cells was also monitored for both WT and Tg females by Q-PCR. WT and Tg mGCs, cGCs, oocytes and whole ovaries were collected at 2, 3, 4 and $\geqslant 6$ months of age and subjected to total RNA extraction. Primers for Umodl1 (forward, 5'-ACGACTTCAGCTACCAGGTGTTCT-3'; reverse, 5'-TGGACTCC CGTCAATGTTCGATGA-3'), PPAR (forward, 5'-GCTGTCATTATTCTCAGTG GAGACCG-3'; reverse, 5'-CTGCAGCAGGTTGTCTTGGATGT-3'), and GAPDH (forward, $5^{\prime}$-CTGGCATTGCTCTCAATGACAACT-3'; reverse, $5^{\prime}$-TTACTCCT TGGAGGCCATGTAGGC-3') were designed. All of the forward and reverse primers were located on different exons so that PCR products derived from genomic versus cDNA templates can be distinguished. Approximately $2 \mu \mathrm{g}$ was reversely transcribed using oligo-dT primers in a total volume of $20 \mu \mathrm{l}$ for each sample. A standard real-time PCR reaction mix was prepared containing the following components: $10 \mu \mathrm{l}$ of SYBR Green PCR master mix (4309155; Applied Biosystems, Foster City, CA, USA), $2 \mu \mathrm{l}$ of cDNA, and $0.2 \mu \mathrm{M}$ of each primer. All samples were run in triplicate on an $\mathrm{ABI} 7900 \mathrm{HT}$ Fast Real-time PCR System (Applied Biosystems) using the following parameters: $10 \mathrm{~min}$ at $95^{\circ} \mathrm{C}$, and 40 cycles of $15 \mathrm{~s}$ at $95^{\circ} \mathrm{C}, 30 \mathrm{~s}$ at $58^{\circ} \mathrm{C}$, and $30 \mathrm{~s}$ at $72^{\circ} \mathrm{C}$. Data retrieval and analysis were performed using software SDS2.3 and Microsoft Excel (Microsoft Corp., Redmond, WA, USA). To normalize the quantity of total mRNA, GAPDH mRNA was amplified in parallel in different tubes. Gene expression levels were calculated after normalization to the housekeeping gene, $G A P D H$, using the comparative $\mathrm{C}_{\mathrm{T}}$ $(\Delta \Delta \mathrm{Ct})$ method. ${ }^{40} \mathrm{mRNA}$ expression data were presented as averages of the fold changes relative to the controls.

Assessment of female fertility. Continuous breeding began at 6 weeks of age. $\operatorname{Tg}(n=13)$ or WT control $(n=24)$ females were cohabitated with FVB WT males. Litter size was documented. Female age indicated in Figure $5 a$ and $b$ was defined as the postnatal days of copulatory plug detection. Morphometric analysis of follicle number at 2, 4 and 6 months of age was performed as previously described with some modifications. ${ }^{41}$ pW224 Tg ( $n=5 /$ stage) and WT or pW209 control $(n=5 /$ stage) ovaries were collected and cryo-sectioned at $8 \mu \mathrm{m}$ thickness. Sections were fixed and stained for $\beta$-galactosidase activity, followed by nuclearfast-red counter staining. Every third section was examined. Primordial follicles (diameter $<60 \mu \mathrm{m}$ ) were excluded from this study to minimize errors in scoring early follicles (as Tg ovaries older than 4 months contain no primordial follicles). All follicles at more advanced stages were counted under an Olympus BX41 microscope.

Histological analysis. $\beta$-Galactosidase (lacZ) staining was performed as previously described. ${ }^{35}$ Ovaries from pW209 Lycat-BAC Tg females were included as the healthy controls (Figure 5). ${ }^{17}$ The pW209 transgene showed an identical expression profile to the endogenous Lycat. More importantly, no functional Lycat protein can be produced from this transgene, thus rendering no defects in the reproductive system.

Accumulation of fat droplets in the degenerating $\mathrm{Tg}$ follicles was visualized by Oilred-O staining. Ovaries from 4-month-old $\mathrm{Tg}$ females $(n=3)$ were collected, embedded into Neg-50, and cut by a cryostat. Pre-treatment of samples and staining were performed as described. ${ }^{42}$ Stained sections were evaluated and photographed with an Olympus BX41 microscope controlled by DP2-BSW software.

Assessment of apoptosis. Paraffin sections of Tg ovaries $(n=5)$ at 4 and 6 months of age were used for in situ analysis of DNA fragmentation by TUNEL. Sections from 6-month-old WT ovaries $(n=3)$ were also included as controls. Labeling of DNA strand breaks was performed using In Situ Cell Death Detection Kit, Fluorescein (11684795910; Roche Applied Science, Indianapolis, IN, USA) and analyzed with a Nikon Eclipse 90i fluorescence microscope (Nikon Instruments, Inc, Melville, NY, USA). Additionally, progression of apoptosis in the $\mathrm{Tg}$ ovaries was analyzed by agarose gel electrophoresis. Ovaries $(n=2$ per stage, pooled) were collected from Tg females aged 2, 4 and 6 months. Genomic DNA from 6-month-old WT ovaries was used as the healthy control. Ovarian samples were digested with Proteinase $\mathrm{K}$ and extracted by the phenol/chloroform method. One tenth of the total DNA extracted from each ovarian sample was loaded on a $1 \%$ agarose gel. Genomic DNA from the rotten liver of a dead mouse was loaded as a necrotic control.

Statistical analysis. Statistical significance was calculated by one-way ANOVA. The unpaired $t$-test was used to compare results between control and sample groups in each assay. $P$ values $<0.05$ were considered to be statistically significant.

\section{Conflict of Interest}

The authors declare no conflict of interest.

Acknowledgements. We would like to thank Drs Jenny Xiang and Huimin Shan at the Genomic Center of Weill Medical College of Cornell University for performing the quantitative PCR assay; and Dr. Geming Lu at Mount Sinai School of Medicine for technical assistance. We are thankful to The Ronald O Perelman and Claudia Cohen Center for Reproductive Medicine, Weill Medical College of Cornell University for funding this work.

1. Richardson SJ, Senikas V, Nelson JF. Follicular depletion during the menopausal transition: evidence for accelerated loss and ultimate exhaustion. J Clin Endocrinol Metab 1987; 65: 1231-1237

2. te Velde ER, Pearson PL. The variability of female reproductive ageing. Hum Reprod Update 2002; 8: 141-154.

3. Nikolaou D, Templeton A. Early ovarian ageing. Eur J Obstet Gynecol Reprod Biol 2004; 113: $126-133$.

4. Burgering BM, Medema RH. Decisions on life and death: FOXO Forkhead transcription factors are in command when PKB/Akt is off duty. J Leukoc Biol 2003; 73: 689-701.

5. Castrillon DH, Miao L, Kollipara R, Horner JW, DePinho RA. Suppression of ovarian follicle activation in mice by the transcription factor Fox03a. Science 2003; 301: 215-218

6. Dong J, Albertini DF, Nishimori K, Kumar TR, Lu N, Matzuk MM. Growth differentiation factor-9 is required during early ovarian folliculogenesis. Nature 1996; 383: 531-535.

7. Yan C, Wang P, DeMayo J, DeMayo FJ, Elvin JA, Carino C et al. Synergistic roles of bone morphogenetic protein 15 and growth differentiation factor 9 in ovarian function. $\mathrm{Mol}$ Endocrinol 2001; 15: 854-866. 
8. Perez GI, Robles R, Knudson CM, Flaws JA, Korsmeyer SJ, Tilly JL. Prolongation of ovarian lifespan into advanced chronological age by Bax-deficiency. Nat Genet 1999; 21: 200-203.

9. Davisson MT, Bechtel LJ, Akeson EC, Fortna A, Slavov D, Gardiner K. Evolutionary breakpoints on human chromosome 21. Genomics 2001; 78: 99-106.

10. Shibuya K, Nagamine K, Okui M, Ohsawa Y, Asakawa S, Minoshima S et al. Initial characterization of an uromodulin-like 1 gene on human chromosome 21q22.3. Biochem Biophys Res Commun 2004; 319: 1181-1189.

11. Nishizaki R, Ota M, Inoko H, Meguro A, Shiota T, Okada E et al. New susceptibility locus for high myopia is linked to the uromodulin-like 1 (UMODL1) gene region on chromosome 21q22.3. Eye 2009; 23: 222-229.

12. Murphy C, Jinich S. Olfactory dysfunction in Down's Syndrome. Neurobiol Aging 1996; 17 : $631-637$.

13. Di Schiavi E, Riano E, Heye B, Bazzicalupo P, Rugarli El. UMODL1/Olfactorin is an extracellular membrane-bound molecule with a restricted spatial expression in olfactory and vomeronasal neurons. Eur J Neurosci 2005; 21: 3291-3300.

14. Peters LM, Belyantseva IA, Lagziel A, Battey JF, Friedman TB, Morell RJ. Signatures from tissue-specific MPSS libraries identify transcripts preferentially expressed in the mouse inner ear. Genomics 2007; 89: 197-206.

15. Markopoulos F, Neubauer FB, Berger T, Scotti AL. Reassembling a system from the sensor to cerebral representation: the olfactory system in vitro. Neuroscience 2008; 156: 1048-1063.

16. Sammeta N, Hardin DL, McClintock TS. Uncx regulates proliferation of neural progenitor cells and neuronal survival in the olfactory epithelium. Mol Cell Neurosci 2010; 45: 398-407.

17. Wang W, Ni L, Yu Q, Xiong J, Liu HC, Rosenwaks Z. Expression of the Lycat gene in the mouse cardiovascular and female reproductive systems. Dev Dyn 2010; 239: 1827-1837.

18. Elvin JA, Yan C, Wang P, Nishimori K, Matzuk MM. Molecular characterization of the follicle defects in the growth differentiation factor 9-deficient ovary. Mol Endocrinol 1999; 13: 1018-1034.

19. Singtripop T, Mori T, Shiraishi K, Park MK, Kawashima S. Age-related changes in gonadotropin, prolactin and growth hormone levels with reference to the development of uterine adenomyosis in female SHN mice. In Vivo 1993; 7: 147-150.

20. Parkening TA, Collins TJ, Smith ER. Plasma and pituitary concentrations of luteinizing hormone, follicle-stimulating hormone and prolactin in aged, ovariectomized CD-1 and C57BL/6 mice. Exp Gerontol 1982; 17: 437-443.

21. van Zonneveld $P$, Scheffer GJ, Broekmans FJ, te Velde ER. Hormones and reproductive aging. Maturitas 2001; 38: 83-91; discussion 92-4.

22. Reynolds J, Keverne EB. The accessory olfactory system and its role in the pheromonally mediated suppression of oestrus in grouped mice. J Reprod Fertil 1979; 57: 31-35.

23. Smith TD, Siegel MI, Bhatnagar KP. Reappraisal of the vomeronasal system of catarrhine primates: ontogeny, morphology, functionality, and persisting questions. Anat Rec 2001; 265: 176-192

24. Foltan R, Sedy J. Behavioral changes of patients after orthognathic surgery develop on the basis of the loss of vomeronasal organ: a hypothesis. Head Face Med 2009; 5: 5 .

25. Kaneko N, Debski EA, Wilson MC, Whitten WK. Puberty acceleration in mice. II. Evidence that the vomeronasal organ isa receptor for the primer pheromone in male mouse urine. Biol Reprod 1980; 22: 873-878.

26. Bellringer JF, Pratt HP, Keverne EB. Involvement of the vomeronasal organ and prolactin in pheromonal induction of delayed implantation in mice. J Reprod Fertil 1980; 59: 223-228.
27. Nishizuka Y, Sakakura T. Thymus and reproduction: sex-linked dysgenesia of the gonad after neonatal thymectomy in mice. Science 1969; 166: 753-755.

28. Michael SD, Taguchi O, Nishizuka Y. Changes in hypophyseal hormones associated with accelerated aging and tumorigenesis of the ovaries in neonatally thymectomized mice. Endocrinology 1981; 108: 2375-2380.

29. Miller ME, Chatten J. Ovarian changes in ataxia telangiectasia. Acta Paediatr Scand 1967; 56: 559-561.

30. Perisic M, Arsenovic-Ranin N, Pilipovic I, Kosec D, Pesic V, Radojevic K et al. Role of ovarian hormones in age-associated thymic involution revisited. Immunobiology 2010; 215: 275-293.

31. Forsberg JG. Neonatal estrogen treatment and its consequences for thymus development, serum level of autoantibodies to cardiolipin, and the delayed-type hypersensitivity response. J Toxicol Environ Health A 2000; 60: 185-213.

32. McMahon HE, Hashimoto O, Mellon PL, Shimasaki S. Oocyte-specific overexpression of mouse bone morphogenetic protein-15 leads to accelerated folliculogenesis and an early onset of acyclicity in transgenic mice. Endocrinology 2008; 149: 2807-2815.

33. Schomberg DW, Couse JF, Mukherjee A, Lubahn DB, Sar M, Mayo KE et al. Targeted disruption of the estrogen receptor-alpha gene in female mice: characterization of ovarian responses and phenotype in the adult. Endocrinology 1999; 140: 2733-2744.

34. Schmidt D, Ovitt CE, Anlag K, Fehsenfeld S, Gredsted L, Treier AC et al. The murine winged-helix transcription factor Foxl2 is required for granulosa cell differentiation and ovary maintenance. Development 2004; 131: 933-942.

35. Wang W, Van De Water T, Lufkin T. Inner ear and maternal reproductive defects in mice lacking the Hmx3 homeobox gene. Development 1998; 125: 621-634.

36. Wang W, Chen X, Xu H, Lufkin T. Msx3: a novel murine homologue of the Drosophila msh homeobox gene restricted to the dorsal embryonic central nervous system. Mech Dev 1996; 58: 203-215.

37. Moore D, Chory J, Ribaudo RK. Isolation and purification of large DNA restriction fragments from agarose gels. Curr Protoc Immunol 2001, Chapter 10: Unit 105.

38. Nelson JF, Felicio LS, Randall PK, Sims C, Finch CE. A longitudinal study of estrous cyclicity in aging C57BL/6J mice: I. Cycle frequency, length and vaginal cytology. Biol Reprod 1982; 27: 327-339.

39. Salustri A, Ulisse S, Yanagishita M, Hascall VC. Hyaluronic acid synthesis by mural granulosa cells and cumulus cells in vitro is selectively stimulated by a factor produced by oocytes and by transforming growth factor-beta. J Biol Chem 1990; 265: 19517-19523.

40. Bookout AL, Mangelsdorf DJ. Quantitative real-time PCR protocol for analysis of nuclear receptor signaling pathways. Nucl Recept Signal 2003; 1: e012.

41. Durlinger AL, Kramer P, Karels B, de Jong FH, Uilenbroek JT, Grootegoed JA et al. Control of primordial follicle recruitment by anti-Mullerian hormone in the mouse ovary. Endocrinology 1999; 140: 5789-5796.

42. Sheehan DCH, Barbara B. Theory and practice of Histotechnology. Battle Press, 1980.

Cell Death and Disease is an open-access journal published by Nature Publishing Group. This work is licensed under the Creative Commons Attribution-NonCommercialShare Alike 3.0 Unported License. To view a copy of this license, visit http://creativecommons.org/licenses/by-nc-sa/3.0/

Supplementary Information accompanies the paper on Cell Death and Disease website (http://www.nature.com/cddis) 